\title{
Research on the viscosity of stabilized emulsions in different pipe diameters using pressure drop and phase inversion
}

\author{
Jose Plasencia ${ }^{1}$, Nathanael Inkson², Ole Jørgen Nydal' $(\bowtie)$ \\ 1. Department of Energy and Process Engineering, Norwegian University of Science and Technology, NO-7491 Trondheim, Norway \\ 2. Siemens Digital Industries Software, Simulation and Test Solutions, Product Development, UK
}

\begin{abstract}
This paper reports experimental research on the flow behavior of oil-water surfactant stabilized emulsions in different pipe diameters along with theoretical and computational fluid dynamics (CFD) modeling of the relative viscosity and inversion properties. The pipe flow of emulsions was studied in turbulent and laminar conditions in four pipe diameters $(16,32,60$, and $90 \mathrm{~mm})$ at different mixture velocities and increasing water fractions. Salt water $(3.5 \% \mathrm{NaCl} w / \mathrm{v}, \mathrm{pH}=7.3)$ and a mineral oil premixed with a lipophilic surfactant (Exxsol D $80+0.25 \% \mathrm{v} / \mathrm{v}$ of Span 80 ) were used as the test fluids. The formation of water-in-oil emulsions was observed from low water fractions up to the inversion point. After inversion, unstable water-in-oil in water multiple emulsions were observed under different flow regimes. These regimes depend on the mixture velocity and the local water fraction of the water-in-oil emulsion. The eddy turbulent viscosity calculated using an elliptic-blending $k-\varepsilon$ model and the relative viscosity in combination act to explain the enhanced pressure drop observed in the experiments. The inversion process occurred at a constant water fraction (90\%) and was triggered by an increase of mixture velocity. No drag reduction effect was detected for the water-in-oil emulsions obtained before inversion.

\author{
Keywords \\ oil-water flow \\ emulsion rheology \\ effective viscosity \\ phase inversion \\ pressure drop \\ Article History \\ Received: 1 July 2020 \\ Revised: 14 December 2020 \\ Accepted: 28 December 2020 \\ Research Article \\ (c) The Author(s) 2021
}

\section{Introduction}

The pipe flow of stable dispersions, also known as emulsions, is commonly found in different applications going from the oil to the food industry. In the oil industry, the emulsification of water-in-oil may occur during crude oil transportation in the presence of produced water. Thus, water starts to disperse into the oil phase when submitted to intense shear rates, and the resultant mixture is usually stabilized by the surface active components present in crude oil. The formation of water-in-oil emulsions may dramatically increase the effective viscosity of the mixture affecting the pressure drop in the flow lines and therefore the production rates. Experimental studies with both stable and unstable oil-water dispersed flow are available in literature. The formation of unstable dispersions is typically achieved by using two immiscible fluids whereas the stabilized emulsions are obtained by the addition of a surface active component in one of the phases. In the literature, pressure drop and phase inversion pipe flow experiments were predominantly performed using unstable oil-water mixtures (Cengel et al.,
1962; Arirachakaran et al., 1989; Nädler and Mewes, 1997; Angeli and Hewitt, 1999; Lovick and Angeli, 2004; Ioannou et al., 2005; Piela et al., 2006; Plasencia and Nydal, 2010), while most of the experimental works on phase inversion with stable emulsions have been done in small stirred vessels (Groeneweg et al., 1998; Salager et al., 2000; Tyrode et al., 2003; Galindo-Alvarez et al., 2011).

The addition of surfactants, which affects the droplet sizes and the stability of the dispersion, reduces the interfacial tension between the two phases promoting emulsification. Hence, the presence of surfactants in the oil phase approaches the behavior of a mineral oil to a real crude oil. Under similar pipe flow conditions droplet sizes appear to be smaller in stable than unstable dispersions. Due to these differences, unstable dispersions generally result in a small increase of pressure drop close to the inversion point that is usually of the order of the single phase oil pressure drop (Plasencia and Nydal, 2010).

In contrast, stable emulsions may present a significant increase of pressure drop that might be dramatically close to the inversion point. The higher stability also has an impact

$\triangle$ ole.j.nydal@ntnu.no 


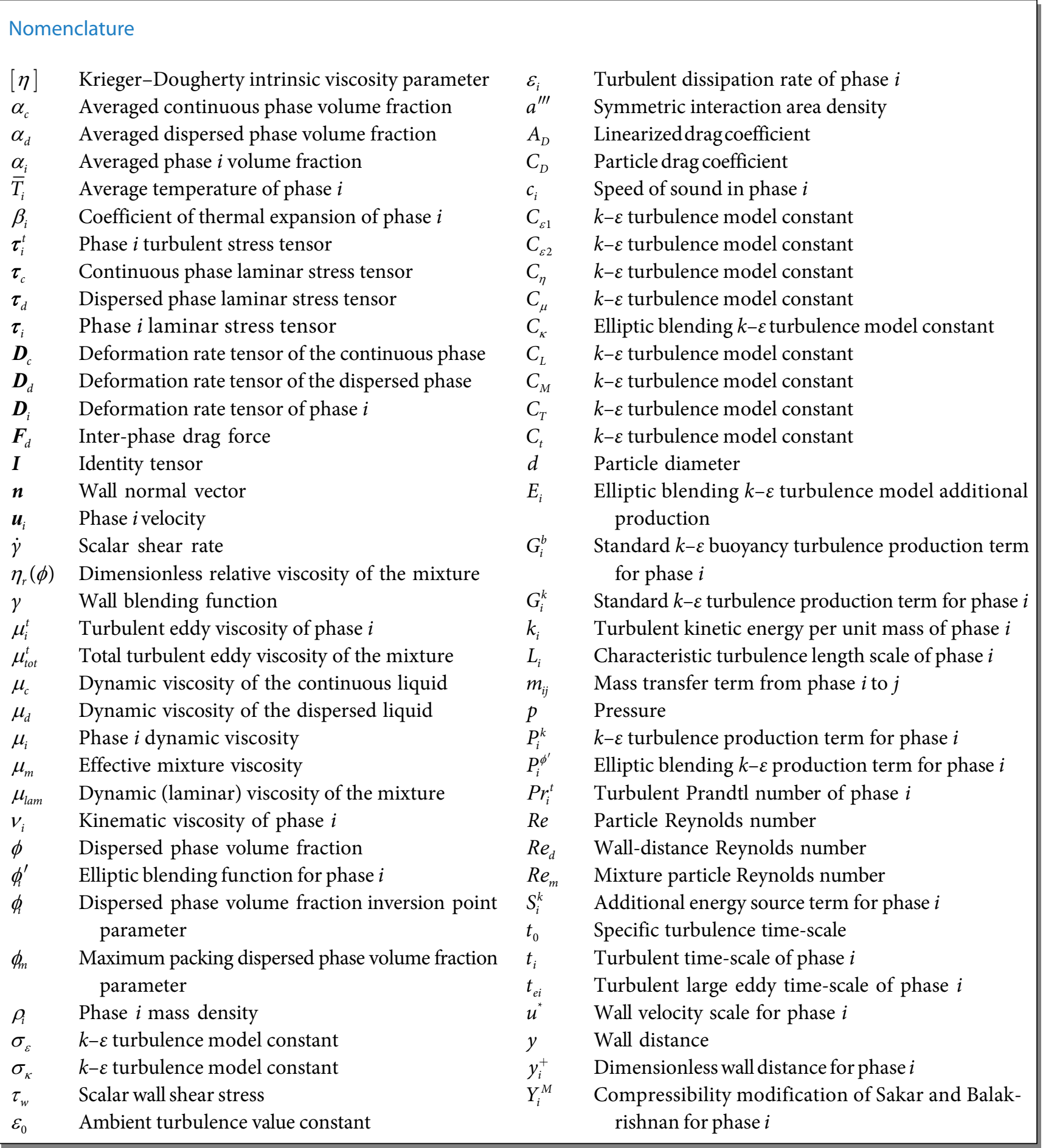

on the phase inversion point by preventing droplet coalescence and thus retarding inversion to higher dispersed phase fractions. A drag reduction effect has also been reported by many authors in oil-water unstable dispersed flows (Pal, 1993, 2007; Groeneweg et al., 1998; Angeli and Hewitt, 1999; Ioannou et al., 2005; Plasencia and Nydal, 2010). This phenomenon has been attributed to the effect of turbulence on droplet breakup and coalescence. It was observed that drag reduction is significantly reduced or non-existent in stable dispersed flow (Pal, 1993). We report an experimental and theoretical study of the flow behavior of water-in-oil surfactant stabilized emulsions in different pipe diameters. A literature review confirmed few studies on the behavior of emulsions under turbulent flow conditions due to the small pipe diameters commonly used, and the high effective viscosities of the emulsions. 
Four different pipe diameters were tested with the aim of studying how water-in-oil emulsions behave under turbulent and laminar conditions. The verification of the existence of a drag reduction effect was also of the interest of this study. Pressure drop measurements were performed in four pipe diameters $(16,32,60$, and $90 \mathrm{~mm})$ for a mixture of salt water $(3.5 \% \mathrm{NaCl} w / \mathrm{v}, \mathrm{pH}=7.3)$ and the mineral oil Exxsol D80. A lipophilic surface active component was premixed with the oil phase $(0.25 \% \mathrm{v} / \mathrm{v}$ of Span 80$)$ to stabilize the emulsion and mimic a real crude oil. The phase inversion process was studied using impedance rings and visual inspection. The type of emulsions formed was also studied by sampling and visualization under the microscope. Droplet sizes were measured in-situ using a focused beam reflectance measurement probe (FBRM).

In order to model stabilized emulsion rheology in Computational Fluid Dynamics (CFD) simulation the authors built upon a formulation using a two-fluid (Euler-Euler) formulation following the work of Ishii and Hibiki (2006). This approach is well established for bubbly flow in air and water, granular flow in air-solid mixtures, and oil and water for pipelines (Lo, 2015).

Relative (mixture) viscosity models were developed for suspensions of particles in a liquid by Krieger and Dougherty (1959) where the relative viscosity depends only upon the volume fraction of the particles. As the concentration of particles increases, an exponential increase in viscosity occurs as the particles start to interact causing moments of hydrodynamic lubrication forces to become large. Eventually jamming occurs at the critical volume fraction (maximum packing) when frictional contact points come into play. With an emulsion however a phase inversion occurs at a critical volume fraction and the effect of maximum packing is not so strong as the droplets are deformable and can coalesce.

The two-phase code was formulated taking the relative viscosity and splitting it between the phases based on an average volume fraction. This has been used to successfully model the effects of shear-induced particle migration in solid suspensions of particles in a liquid (Inkson et al., 2017). Earlier work studying crude-oil and salt water stabilized emulsions which have properties similar to hard particle suspensions has shown that this approach has been successful in modeling the pressure drop in pipe flow (Inkson et al., 2014).

In order to model the turbulence in the flow we chose an "elliptic blending" $k-\varepsilon$ model of Billard and Laurence (2012) which is a modern and robust Reynolds Averaged Navier Stokes (RANS) model which quantifies but averages out turbulent velocity fluctuations. The elliptic blending turbulence model solves transport equations for the turbulent kinetic energy $k$ (the turbulent kinetic energy per unit mass of the velocity fluctuations) and $\varepsilon$ the turbulent dissipation rate (which is the rate that $k$ is turned into thermal energy); the normalized (reduced) wall-normal stress component $\phi^{\prime}$, and the elliptic blending factor $\alpha^{\prime}$ in order to determine the turbulent eddy viscosity. The concept of elliptic relaxation was proposed by Durbin (1993) for Reynolds-stress models. The initial model required the solution of six additional transport equations, but this number was later reduced to a single additional equation. The model was later simplified by Manceau and Hanjalić (2002) and SDIS (2020).

\section{Theory}

\subsection{Constitutive model}

The pressure drop in a pipe of diameter $D$, across length $\Delta L$ is related to the wall shear stress, $\tau_{w}$ by

$$
\Delta p=\frac{4 \Delta L \tau_{w}}{D}
$$

where the wall shear stress is related to the fluid mixture effective viscosity, $\mu_{m}$ as

$$
\tau_{w}=\mu_{m} \dot{\gamma}_{w}
$$

where $\dot{\gamma}_{w}$ is the strain rate at the wall. For a homogeneous emulsion or suspension the laminar mixture viscosity is

$$
\mu_{\text {lam }}=\eta_{r}(\phi) \mu_{c}
$$

where $\eta_{r}(\phi)$ is the dimensionless relative viscosity and $\mu_{c}$ is the dynamic viscosity of the continuous phase liquid.

The Krieger-Dougherty model (Krieger and Dougherty, 1959) was one of the first numerical descriptions of the relative viscosity of a suspension of hard particles of volume fraction $\phi$ :

$$
\eta_{r}(\phi)=\left(1-\frac{\phi}{\phi_{m}}\right)^{-[\eta] \phi_{m}}
$$

where $[\eta]$ is the intrinsic viscosity parameter. $[\eta]=2.5$ for spherical particles and $\phi_{m}$ is the maximum critical packing fraction $\left(\phi_{m}=0.645\right)$ for the random close packing of monodisperse hard spheres.

For a stabilized emulsion we modified the form of the Krieger-Dougherty relative viscosity model (Inkson et al., 2014) to reflect the physics of emulsions, namely: a) Emulsions will invert at a critical volume fraction $\phi_{2}$ and $b$ ) that the value of maximum packing can exceed unity when using Eq. (4). Therefore we introduce a new pre-factor to the maximum packing which we denote as the emulsion calibration coefficient, $r$.

The relative viscosity of a stabilized emulsion with an inversion volume fraction of $\phi_{t}$ is therefore given by 


$$
\eta_{r}(\phi)= \begin{cases}\left(1-\frac{\phi}{r \phi_{m}}\right)^{-r[\eta] \phi_{m}}, & \phi \leq \phi \\ \left(1-\frac{1-\phi}{r_{i} \phi_{m i}}\right)^{-r_{i}[\eta] \phi_{\phi_{i i}}}, & \phi>\phi\end{cases}
$$

where $r$ and $r_{i}$ are the emulsion calibration coefficients which allow the emulsions to shift the "maximum packing" to a higher value reflecting the softer behavior of the droplets compared to a suspension of hard solid particles, as it becomes meaningless to have a maximum packing fraction above unity. Thus we introduce a new parameter and leave the maximum packing (of a suspension of spheres) unchanged.

As $r \rightarrow 1$ we regain solid particle behaviour. As $r \rightarrow \infty$, $\eta_{r} \rightarrow 1$ and we regain the Newtonian viscosity of the continuous liquid. In between we get a mixture viscosity far higher than the sum of the Newtonian fluids that comprise the emulsion which represents the surface tension in the droplets, the interaction between them including jamming and large hydrodynamic forces present in the continuous liquid between the droplets. Ultimately the jamming effect gives way to a phase inversion when the dispersed phase volume fraction exceeds the critical limit. Figure 1 shows how the curve of relative viscosity switches to the inverted curve around the inversion point $\phi_{1} \cdot r_{i}$ is the inverted calibration coefficient reflecting that the inverted curve can have a different asymptote of $r_{i} \phi_{m}$ as we have seen with crude oil in salt water emulsions (Plasencia et al., 2013; Inkson et al., 2014).

These models are implemented in the CFD software Simcenter 3D STAR-CCM+ and extensive validation of the multiphase approach has been performed on suspension of particles in liquids (Inkson et al., 2017). In the current

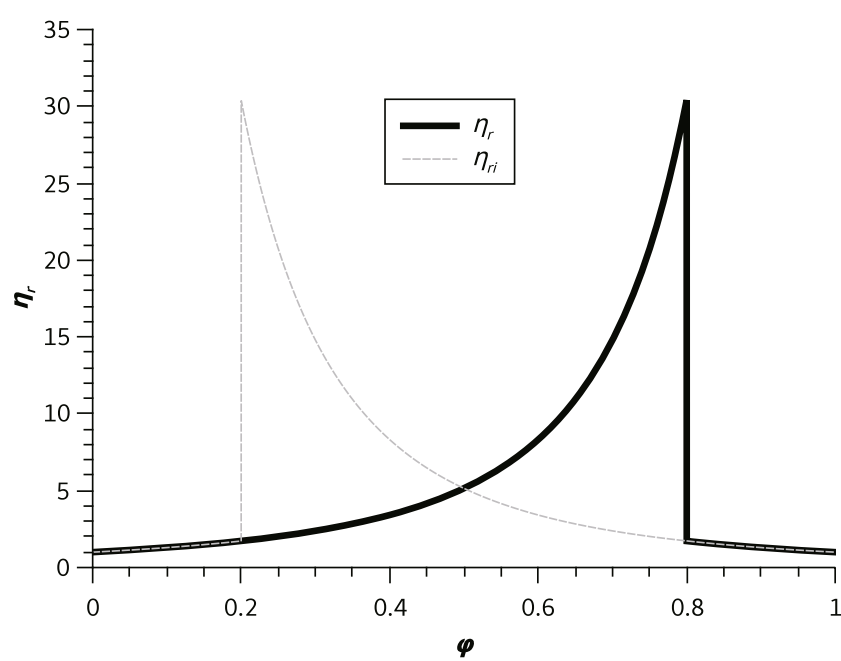

Fig. 1 Black curve shows the relative viscosity of a stabilized water-in-oil emulsion which has an inversion volume fraction of $\phi_{i}=0.8, r=r_{i}=1.7$. At higher volume fractions the model switches to the inverted oil-in-water curve (shown in gray). implementation the value of the inversion point is inputted by the user.

The mixture viscosity described above is for laminar flow; however when we have turbulent flow we also have to factor in the eddy viscosity that is generated by large eddys transporting momentum across layers of fluid. The total effective mixture viscosity is given by the sum of the total laminar viscosity and the total eddy viscosity:

$$
\mu_{m}=\mu_{\text {lam }}+\mu_{\text {tot }}^{t}
$$

Substituting Eq. (3) gives

$$
\mu_{m}=\eta_{r}(\phi) \mu_{c}+\mu_{t o t}^{t}
$$

where the total eddy turbulent viscosity, $\mu_{t o t}^{t}$ is the sum of the phase eddy turbulent viscosities, $\mu_{i}^{t}$ :

$$
\mu_{t o t}^{t}=\sum_{i=1}^{2} \alpha_{i} \mu_{i}^{t}
$$

In the simulations $\mu_{i}^{t}$ is calculated from the turbulence model, see Eq. (22). Note that for dispersed phase volume fractions above the inversion point volume fraction the concepts of dispersed and continuous phases are reversed so Eq. (3) becomes

$$
\mu_{\text {lam }}=\eta_{r}(\phi) \mu_{d}
$$

\subsection{Multi-fluid model}

\subsubsection{Continuity and momentum}

The Euler-Euler methodology defines phases as interpenetrating continua coexisting in the domain (Ishii and Hibiki, 2006). Each phase has its own distinct velocity, temperature, and viscosity amongst other quantities. Conservation equations are solved for each phase with additional closure laws to model the interactions between the phases. This approach is well established for bubbly flow in air and water, granular flow in air-solid mixtures, and oil and water for pipelines (Lo, 2015). We solve the continuity and momentum equation for each phase, $i$. For the rest of the paper we use the suffix $o$ for the continuous liquid oil phase and $w$ for the dispersed water droplet phase. The conservation equations for mass and momentum take the following form for phase $i$ :

$$
\frac{\partial}{\partial t}\left(\alpha_{i} \rho_{i}\right)+\nabla \cdot\left(\alpha_{i} \rho_{i} \boldsymbol{u}_{i}\right)=\mathbf{0}
$$

where $\rho_{i}$ is the phase density and $\boldsymbol{u}_{i}$ is phase velocity.

$$
\begin{aligned}
& \frac{\partial}{\partial t}\left(\alpha_{i} \rho_{i} \boldsymbol{u}_{i}\right)+\nabla \cdot\left(\alpha_{i} \rho_{i} \boldsymbol{u}_{i} \boldsymbol{u}_{i}\right) \\
& \quad=-\alpha_{i} \nabla p+\alpha_{i} \rho_{i} \boldsymbol{g}+\nabla \cdot\left(\boldsymbol{\tau}_{i}+\boldsymbol{\tau}_{i}^{t}\right) \pm \boldsymbol{F}_{i}
\end{aligned}
$$


where $\tau_{i}^{t}$ is the stress tensor from the turbulence model and $\boldsymbol{F}_{i}$ are the interaction forces between the phases and are comprised of contributions from drag force, lift force, virtual mass force, and wall lubrication force. In this work we only considered the effect of drag force, $\boldsymbol{F}_{i}=\boldsymbol{F}_{d}$. For a Newtonian fluid the phase stress tensor is

$$
\boldsymbol{\tau}_{i}=2 \alpha_{i} \mu_{i}\left[\boldsymbol{D}_{i}-\frac{1}{3}\left(\nabla \cdot \boldsymbol{u}_{i}\right) \boldsymbol{I}\right]
$$

where $\mu_{i}$ is the phase dynamic viscosity. Note that traditionally the dispersed particle (droplet) phase volume fraction is $\phi$ which in multiphase notation is now $\alpha_{d}$. In order to split this stress between the phases, we make the assumption that the averaged sum of the stress of the continuous phase and the stress of the dispersed phase is equal to the mixture stress, which is justified in that the relative viscosity accounting for the mixture appears equally in both terms (weighted by the respective volume fraction). The continuous liquid phase stress is

$$
\boldsymbol{\tau}_{c}=2 \alpha_{c} \mu_{c}\left[\eta_{r}\left(\alpha_{d}\right) \boldsymbol{D}_{c}-\frac{1}{3}\left(\nabla \cdot \boldsymbol{u}_{c}\right) \boldsymbol{I}\right]
$$

and the dispersed liquid phase stress is

$$
\boldsymbol{\tau}_{d}=2 \alpha_{d}\left[\mu_{c} \eta_{r}\left(\alpha_{d}\right) \boldsymbol{D}_{d}-\frac{1}{3} \mu_{d}\left(\nabla \cdot \boldsymbol{u}_{d}\right) \boldsymbol{I}\right]
$$

The phase turbulent stress is calculated using the Boussinesq approximation:

$$
\boldsymbol{\tau}_{i}^{t}=2 \alpha_{i} \mu_{i}^{t}\left[\boldsymbol{D}_{i}-\frac{1}{3}\left(\nabla \cdot \boldsymbol{u}_{i}\right) \boldsymbol{I}\right]
$$

where $\mu_{i}^{t}$ is the phase turbulent eddy viscosity. This completes the formulation of the stress tensors.

\subsubsection{Dragforce}

We define the inter-phase drag:

$$
\boldsymbol{F}_{D}=A_{D}\left|\Delta \boldsymbol{v}_{r}\right|
$$

where $\left|\Delta \boldsymbol{v}_{r}\right|$ is the relative slip velocity between phases:

$$
\left|\Delta \boldsymbol{v}_{r}\right| \equiv\left|\boldsymbol{u}_{c}-\boldsymbol{u}_{d}\right|
$$

The direction of the drag force added to the momentum equation (10) is opposite to the drag of the other phase.

The linearized drag coefficient, $A_{D}$ is given by

$$
A_{D}=\frac{a^{\prime \prime \prime}}{8} \rho_{c}\left|\Delta v_{r}\right| C_{D}
$$

where $a^{\prime \prime \prime}$ is the symmetric interaction area density and is defined as

$$
a^{\prime \prime \prime}=\frac{6 \alpha_{c} \alpha_{d}}{d}
$$

and $d$ is the particle diameter. The drag coefficient, $C_{D}$ is a the drag coefficient for particles. The Schiller-Naumann drag (for particles in a Newtonian fluid) (Schiller and Naumann, 1935) is given as

$$
C_{D}=\frac{24}{R e}\left(1+R e^{0.681}\right)
$$

We take a novel approach of modifying the drag to account for the increase in viscosity of the mixture by including the relative viscosity (Inkson et al., 2017) so that the mixture single particle Reynolds number is

$$
R e_{m}=\frac{\rho_{c}\left|\Delta v_{r}\right| d}{\mu_{c} \eta_{r}}=\frac{R e}{\eta_{r}}
$$

where $R e$ is the Reynolds number for a particle in the Newtonian continuous liquid phase and $d$ is the particle diameter. The modified drag for a suspension is now

$$
C_{D}=\frac{24 \eta_{r}}{\operatorname{Re}}\left[1+\left(\operatorname{Re} / \eta_{r}\right)^{0.681}\right]
$$

This modification of drag has been shown to effectively slow the settling of the particles and is equivalent to the sedimentation function in Stokes flow. We limit the minimum value of $R e$ to avoid the singularity in the function. In multiphase flow this has been traditionally handled with a correction to the drag to account for the presence of the other particles (Richardson and Zaki, 1997; Rusche and Issa, 2000). However in the context of rheological models it is the relative viscosity that accounts for the presence of the network of particles that increases the drag. This observation that the mixture viscosity replaces the solvent viscosity of the continuous suspending liquid has been observed previously by Lee et al. (1999) when considering the increase in Peclet number around the onset of shear-thickening of silica suspensions.

\subsection{Turbulence model}

\subsection{1 k-ع models}

The original $k-\varepsilon$ model was derived by Jones and Launder (1972) which solves two transport equations for $k$, the turbulent kinetic energy per unit mass of the velocity fluctuations and $\varepsilon$ the turbulent dissipation rate which is the rate that $k$ is turned into thermal energy.

The elliptic blending turbulence model adds additional two equations for the normalized (reduced) wall-normal stress component $\phi^{\prime}$ and the elliptic blending factor $\alpha^{\prime}$ giving improved solutions over the original model. 
The phase turbulent eddy viscosity, $\mu_{i}^{t}$ is modeled as

$$
\mu_{i}^{t}=\rho_{i} C_{\mu} \phi_{i} k_{i} \min \left(t_{i}, \frac{C_{T}}{\sqrt{3} C_{\mu} \phi_{i} \dot{\gamma}_{i}}\right)
$$

where $C_{\mu}, C_{T}$ are model coefficients and the turbulent time-scale is

$$
t_{i}=\sqrt{t_{e i}^{2}+C_{t}^{2} \frac{v_{i}}{\varepsilon_{i}}}
$$

where $t_{e i}=k_{i} / \varepsilon_{i}$ is the phase large eddy time scale and $v_{i}$ is the kinematic viscosity of phase $i$.

The finite volume method integrates material quantities over the volume of a cell so that divergence terms are converted into surface integrals using the divergence theorem.

The transport equation for energy, $k$ of phase $i$ is computed as

$$
\begin{aligned}
& \frac{\partial}{\partial t}\left(\alpha_{i} \rho_{i} k_{i}\right)+\nabla \cdot\left(\alpha_{i} \rho_{i} k_{i} \overline{\boldsymbol{v}}_{i}\right)=\alpha_{i} \nabla \cdot\left[\left(\frac{\mu_{i}}{2}+\frac{\mu_{i}^{t}}{\sigma_{k}}\right) \nabla k_{i}\right] \\
& \quad+\alpha_{i} P_{i}^{k}-\alpha_{i} \rho_{i}\left(\varepsilon_{i}-\varepsilon_{0}\right)+\alpha_{i} S_{i}^{k} \\
& \quad+\sum_{i \neq j}\left(m_{i j} k_{j}^{(i j)}-m_{j i} k_{i}\right)
\end{aligned}
$$

where $\sigma_{k}$ is a model coefficient, $P_{i}^{k}$ is a turbulence production term, $S_{i}^{k}$ are additional source terms (zero in this work), and $m_{i j}$ quantifies mass transfer effects between the phases (zero in this work).

The transport equation for the turbulent dissipation rate is given by

$$
\begin{aligned}
& \frac{\partial}{\partial t}\left(\alpha_{i} \rho_{i} \varepsilon_{i}\right)+\nabla \cdot\left(\alpha_{i} \rho_{i} \varepsilon_{i} \overline{\boldsymbol{v}}_{i}\right)=\alpha_{i} \nabla \cdot\left[\left(\frac{\mu_{i}}{2}+\frac{\mu_{i}^{t}}{\sigma_{\varepsilon}}\right) \nabla \varepsilon_{i}\right] \\
& \quad+\alpha_{i}\left[\frac{1}{t_{i}^{e}} C_{\varepsilon 1} P_{i}^{\varepsilon}-C_{\varepsilon 2} \rho_{i}\left(\frac{\varepsilon_{i}}{t_{i}^{e}}-\frac{\varepsilon_{0}}{t_{i}^{0}}\right)+S_{i}^{\varepsilon}\right] \\
& \quad+\sum_{i \neq j}\left(m_{i j} \varepsilon_{j}^{(i j)}-m_{j i} \varepsilon_{i}\right)
\end{aligned}
$$

where $\sigma_{\varepsilon}, C_{\varepsilon 1}, C_{\varepsilon 2}$ are model coefficients.

The transport equation for the normalized (reduced) wall-normal stress component $\phi^{\prime}$ is

$$
\frac{\partial}{\partial t}\left(\rho_{i} \phi_{i}^{\prime}\right)+\nabla \cdot\left(\rho_{i} \phi_{i}^{\prime} \overline{\boldsymbol{u}}_{i}\right)=\nabla \cdot\left[\left(\frac{\mu_{i}}{2}+\frac{\mu_{i}^{t}}{\sigma_{k}}\right) \nabla \phi_{i}\right]+P_{\phi^{\prime}}+S_{\phi^{\prime}}
$$

The equation for elliptic blending factor $\alpha^{\prime}$ is

$$
\nabla \cdot\left(L_{i}^{2} \nabla \alpha_{i}^{\prime}\right)=\alpha_{i}^{\prime}-1
$$

where $L_{i}$ is the characteristic phase turbulent length scale calculated as

$$
L_{i}=C_{L} \sqrt{\frac{k_{i}^{3}}{\varepsilon_{i}^{2}}+C_{\eta_{i}}^{2} \sqrt{\frac{v_{i}^{3}}{\varepsilon_{i}}}}
$$

where $C_{L}$ and $C_{\eta}$ are model coefficients. $\varepsilon_{0}$ is the ambient turbulence value in the source terms that counteracts turbulence decay. The specific time-scale $t_{0}$ is

$$
t_{0}=\max \left(\frac{k_{i}}{\varepsilon_{i}}, C_{t} \sqrt{\frac{v_{i}}{\varepsilon_{0}}}\right)
$$

For the elliptic blending model the energy production term is

$$
P_{i}^{k}=G_{i}^{k}+G_{i}^{b}+Y_{i}^{M}
$$

where the production terms are the same as the standard $k-\varepsilon$ model as follows; $G_{i}^{k}$ is turbulent production:

$$
G_{i}^{k}=\mu_{i}^{t} \dot{\gamma}_{i}^{2}-\frac{2}{3} \rho_{i} k_{i} \nabla \cdot \overline{\boldsymbol{v}}_{i}-\frac{2}{3} \mu_{i}^{t}\left(\nabla \cdot \overline{\boldsymbol{v}}_{i}\right)^{2}
$$

where $\dot{\gamma}_{i}$ is the phase shear strain-rate, $G_{i}^{b}$ is buoyancy production:

$$
G_{i}^{b}=\beta_{i} \frac{\mu_{i}^{t}}{P r_{i}^{t}} \nabla \bar{T}_{i} \cdot g
$$

where $\beta_{i}$ is the coefficient of thermal expansion of phase $i, \operatorname{Pr}_{i}^{t}$ is the turbulent Prandtl number of phase $i, \bar{T}_{i}$ is the average temperature of phase $i$, and $\boldsymbol{g}$ is the vector of acceleration due to gravity.

Finally $Y_{i}^{M}$ is the compressibility modification of Sakar and Balakrishnan (1990):

$$
Y_{i}^{M}=\frac{\rho_{i} C_{M} k_{i} \varepsilon_{i}}{c_{i}^{2}}
$$

where $C_{M}$ is a model coefficient and $c_{i}$ is the speed of sound.

The elliptic blending dissipation production is given by

$$
P_{i}^{\varepsilon}=G_{i}^{k}+C_{\varepsilon 3} G_{i}^{b}+\frac{1}{C_{\varepsilon 1}} E_{i}
$$

where the additional production is

$$
E_{i}=C_{k}\left(1-\alpha_{i}^{3}\right) v_{i} \mu_{i}^{t} \frac{k_{i}}{\varepsilon_{i}}\left[\nabla \cdot\left(\left|2 \boldsymbol{D}_{i} \boldsymbol{n}\right| \boldsymbol{n}\right)\right]^{2}
$$

where $\boldsymbol{D}$ is the deformation rate tensor, $C_{k}$ is a model coefficient, and $\boldsymbol{n}$ is the wall normal.

The elliptic blending model has an additional production term for $\phi^{\prime}$ given by

$$
P_{i}^{\phi^{\prime}}=\frac{\phi_{i}^{\prime}}{k_{i}}\left(G_{i}^{k}+G_{i}^{b}\right)+\rho_{i}\left(1-\alpha^{\prime 3}\right) f_{w}+\rho_{i} \alpha^{\prime 3} f_{h}
$$

where $f_{w}=-\frac{\phi_{i}^{\prime}}{2 t_{i}^{e}}$ and 


$$
f_{h}=-\frac{1}{t_{i}}\left(C_{1}-1+C_{2} \frac{G_{i}^{k}+G_{i}^{b}}{\rho_{i} \varepsilon_{i}}\right)\left(\phi_{i}^{\prime}-\frac{2}{3}\right)
$$

\subsubsection{Wall treatment}

The dimensionless value of the distance from the wall, $y^{+}$is an important quantity to model the velocity close to the wall, with a value of order unity meaning that you can fully resolve the boundary viscous sub-layer. $y^{+}$is defined for phase $i$ as

$$
y_{i}^{+}=\frac{y \rho_{i} u^{*}}{\mu_{i}}
$$

where $y$ is the distance from the wall and $u_{i}^{*}$ provides the velocity scale between the viscous sub-layer, thorough the buffer layer to the log-layer:

$$
u_{i}^{*}=\gamma \sqrt{\frac{\mu_{i}\left|\boldsymbol{u}_{i}^{t}\right|}{\rho_{i} y}}+(1-\gamma) C_{\mu}^{1 / 4} k_{i}^{1 / 2}
$$

where $y$ is the blending function defined as

$$
\gamma=\exp \left(\frac{-R e_{d}}{11}\right)
$$

and $R e_{d}$ is the wall-distance Reynolds number defined as

$$
R e_{d}=\frac{\sqrt{k} y}{v_{i}}
$$

\subsection{Finite volume method}

The code is built using discrete equations using the finite volume method and the Phase-Coupled SIMPLE (PC-SIMPLE) algorithm (Vasquez and Ivanov, 2000; SDIS, 2020) which is in turn based on the well known SIMPLE (Semi-Implicit Method for Pressure-Linked Equations) algorithm first proposed by Patankar and Spalding (Patankar and Spalding, 1972; Patankar, 1990). This formulation is explained in further detail in Inkson et al. (2017).

\subsection{Pressure drop}

\subsubsection{Determination of laminar or turbulent flow}

Emulsions are well dispersed liquid-liquid systems. As such emulsions can be modeled using the homogeneous model which uses the single phase flow equations with averaged mixture properties. For both laminar and turbulent conditions pressure drop in a pipe is obtained from the momentum balance as

$$
-\frac{\mathrm{d} p}{\mathrm{~d} l}=f_{m} \frac{\rho_{m} U_{m}^{2}}{2 D}
$$

where $\mathrm{d} p / \mathrm{d} l$ is the pressure drop, $f_{m}$ is the Darcy friction factor of the mixture, $\rho_{m}$ is the mixture density, $U_{m}$ is the mixture superficial velocity, $D$ is the pipe diameter, $\mu_{m}$ is the mixture effective viscosity, and $R e_{m}$ is the Reynolds number of the mixture. The friction factor of the mixture is calculated for laminar flow as

$$
f_{m}=\frac{64}{R e_{m}}
$$

and for turbulent flow as

$$
f_{m}=0.316 R e_{m}^{-0.25}
$$

where the Reynolds number for the mixture is

$$
R e_{m}=\frac{\rho_{m} U_{m} D}{\mu_{m}}
$$

We can identify if we are in the laminar or turbulent regime by starting with the simplified momentum balance equation (41) and substituting Eqs. (42) and (43) revealing for laminar flow:

$$
-\frac{\mathrm{d} p}{\mathrm{~d} l}=\left(32 \frac{\mu_{m}}{D^{2}}\right) U_{m}
$$

and for turbulent flow

$$
-\frac{\mathrm{d} p}{\mathrm{~d} l}=\left(0.158 \frac{\rho_{m}^{\frac{3}{4}} \mu_{m}^{\frac{1}{4}}}{D^{\frac{5}{4}}}\right) U_{m}
$$

\section{Experiments}

\subsection{Fluid properties}

Two phase liquid-liquid experiments were carried out using salt water and a mineral oil. The aqueous phase used in the experiments was a mixture of tap water and $3.5 \% \mathrm{w} / \mathrm{v}$ of $\mathrm{NaCl}$ (with a $\mathrm{pH}$ of 7.3). The oil phase was a mixture of the mineral oil Exxsol D80 and 0.25\% in volume of the lipophilic surfactant Span 80 , by Fluka $(\mathrm{HLB}=4.3)$. The addition of Span 80 in the oil phase reduces the oil-water interfacial tension and induces the formation of water-in-oil stable emulsions. The physical properties of the fluids used in the experiments are shown in Table 1.

Table 1 Fluid properties

\begin{tabular}{lcc}
\hline \multicolumn{1}{c}{ Fluid } & Density $\left(\mathrm{kg} \cdot \mathrm{m}^{-3}\right)$ at $20{ }^{\circ} \mathrm{C}$ & Viscosity $(\mathrm{Pa} \cdot \mathrm{s})$ \\
\hline Salt water & 1023 & 0.001 \\
Exxsol D80 & 815 & 0.0018 \\
\hline Interfacial tension $\left(20^{\circ} \mathrm{C}\right)$ & \\
Salt water - Exxsol D80 & $36 \mathrm{mN} / \mathrm{m}$ \\
Salt water - Exxsol D80 $+0.25 \% \mathrm{v} / \mathrm{v}$ Span 80 & $2.6 \mathrm{mN} / \mathrm{m}$ \\
\hline
\end{tabular}




\subsection{Experimental set-up}

Experiments were performed at the Multiphase Flow Laboratory, NTNU (Norwegian University of Science and Technology). The experimental set-up used is presented in Fig. 2. It consists of two separated flow loops. Acrylic pipes with internal diameters of 32,60, and $90 \mathrm{~mm}$ with a length of $15 \mathrm{~m}$ were tested using a medium scale flow loop. These pipes were mounted in parallel and connectors were switched from one to the other for their respective use. A $4 \mathrm{~m}$ long acrylic pipe with an internal diameter of $16 \mathrm{~mm}$ was connected to a separated smaller flow loop. In both cases the pipes were installed in the horizontal position. In the larger test section a $500 \mathrm{~L}$ tank was used to introduce the fluids. This tank was kept at atmospheric pressure and is connected to the suction of the pumps. Two positive displacement low shear pumps (Eccentric helical rotor pumps by Bornemann) were used to circulate the fluid. A small pump was used to reach flow rates up to $5 \mathrm{~m}^{3} / \mathrm{h}$ while a large pump was capable to operate between 5 and $28 \mathrm{~m}^{3} / \mathrm{h}$. Both pumps were connected to a frequency converter making it possible to control the flow rates. From the tank, the fluid was pumped through a steel pipe where a Coriolis flow meter is located to finally enter to the main test section. In the main test section $15 \mathrm{~m}$ long acrylic pipes with different pipe internal diameters $(90,60$, and $32 \mathrm{~mm}$ ID) were used for the measurements. Two differential pressure transducers were used for the ranges of $0-1 \mathrm{kPa}$ and $0-15 \mathrm{kPa}$. Pressure drop was measured over a pipe distance of 1.0, 1.2, and $1.6 \mathrm{~m}$ for the pipe diameters of 90,60 , and $32 \mathrm{~mm}$ respectively. The pressure taps were located at $12 \mathrm{~m}$ downwards from the entrance of the acrylic pipes. Plastic hoses filled with water were used to connect the pipe pressure taps with the differential pressure transducer cell. A small tank filled with water located at a height of $3 \mathrm{~m}$ was used as a loading system to keep the pressure hoses filled with clean water. This loading tank was connected to the pressure hoses through a valve that was opened prior to the pressure drop measurements to remove any oil entrainment which can affect the measurements. With the pressure hoses filled with clean water, the loading valve was closed to proceed with the measurements. Conductance probes were used in two different positions along the acrylic pipes. They consist of two parallel electrode rings that have the same internal diameter as the acrylic pipe. They were located at 8 and $12 \mathrm{~m}$ from the acrylic pipe inlet and were used to detect the presence of water on the pipes wall. Additionally, a traversing gamma densitometer was used in the $60 \mathrm{~mm}$ pipe. The purpose of this instrument was to study the distribution of the dispersed phase in the pipe cross section as a function of water cut.

Droplet size distributions at different water fractions and superficial velocities were also studied. In-situ chord length distributions were measured using the focused beam reflectance technique (FBRM probe from Mettler Toledo). The FBRM probe was mounted at $45^{\circ}$ from the horizontal as recommended by the supplier. Studies show that the FBRM technique tends to underestimate the actual droplet size (Boxall et al., 2009; Maaß et al., 2011).

However, this technique gives a good idea about the size distribution of droplets and it is very useful to track on-line in-situ droplet size variations in a process. Finally, the fluid temperature was measured using thermocouples located close to the each pipe outlet. The $16 \mathrm{~mm}$ ID pipe was connected to a smaller flow loop. In this test section a $30 \mathrm{~L}$ tank was used to introduce the fluids in the system.

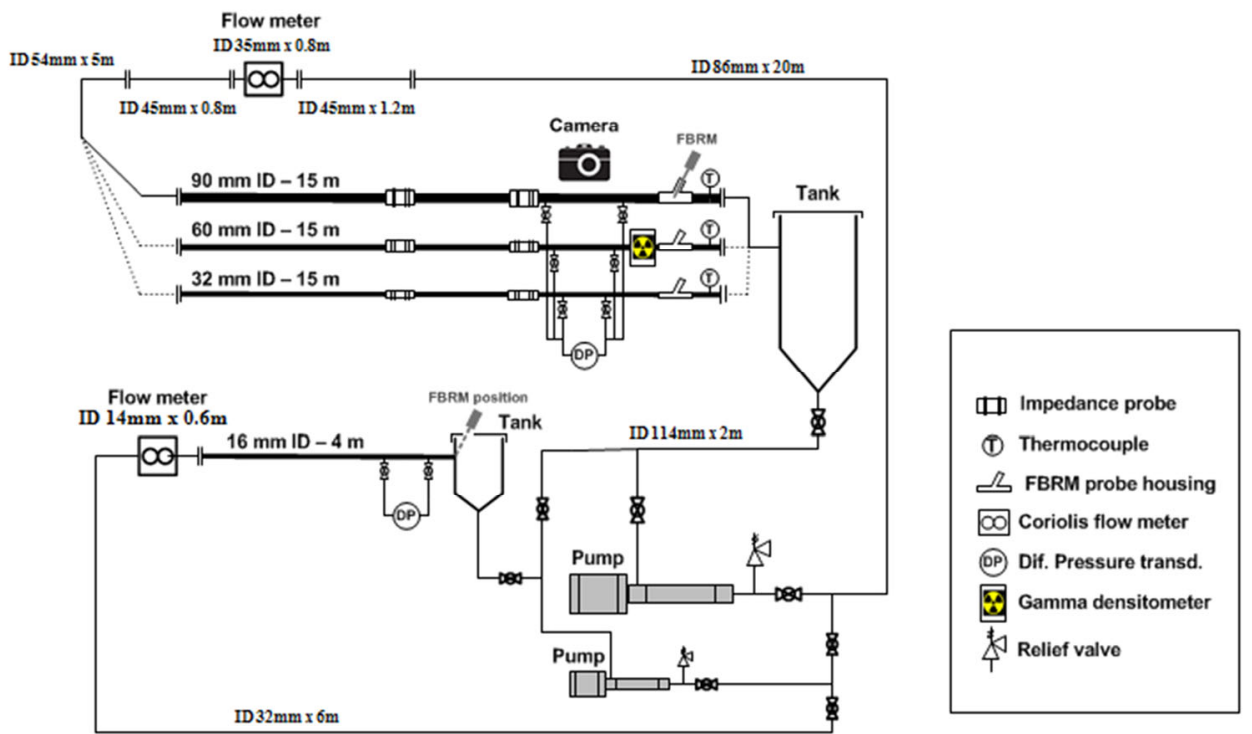

Fig. 2 Experimental set-up. 
For this small pipe only the small positive displacement pump was used. This pump was capable of reaching the needed flow rates. The fluid was pumped from the tank through a $6 \mathrm{~m}$ long flexible hose with $32 \mathrm{~mm}$ internal diameter. Downstream of the flexible pipe a Coriolis flow meter and then the $4 \mathrm{~m}$ long acrylic pipe were connected. In the acrylic pipe, pressure taps were located $3 \mathrm{~m}$ from the inlet where pressure drop was measured over a distance of $0.5 \mathrm{~m}$. In this smaller flow loop, the FBRM probe was mounted at the pipe outlet. Impedance rings were not used in this pipe diameter. The pumps and instrumentation in both flow loops were connected to a labview program to facilitate the flow loop control and data acquisition.

\subsection{Experimental procedure}

Experiments were carried out starting from single phase oil (with 0.25 vol\% of Span 80 ) and increasing the volume fraction of salt water. In the large flow loop the experiments were initiated by introducing single phase oil in the tank. The large flow loop was initially connected to the $90 \mathrm{~mm}$ pipe. With the fluid in the tank, the pump was turned on setting the frequency controller to start at the lowest desired mixture velocity. Once the steady state condition was reached the measurements were performed and the pump frequency was set to get the next mixture velocity for a new measurement. Following this procedure the mixture velocity was increased to perform the measurements in the velocity range desired. After completing the measurement in the $90 \mathrm{~mm}$ pipe, the flow loop was connected to the $60 \mathrm{~mm}$ pipe repeating the same measurement procedure. Finally, the $32 \mathrm{~mm}$ pipe was connected and the procedure was repeated until the measurements were completed. Then, the $32 \mathrm{~mm}$ pipe was replaced by the $90 \mathrm{~mm}$ pipe to start the experiments at a higher water cut. The next water cut was obtained by removing oil from the tank and adding the same volume of salt water. The removed and added volumes were calculated to match the desired water cut. Salt water was introduced in the tank without any type of mixing procedure. With the $90 \mathrm{~mm}$ pipe back in the loop the pump was again turned on at the lowest mixture velocity. The fluids were circulated until a homogeneous dispersion of water-in-oil was obtained. The water fraction in the emulsion was checked by measuring the density of the mixture with the Coriolis flow meter. It was always verified that the density calculated based on the volume fraction of each phase introduced in the tank matches the flow meter density measurements (no slip). Typically between 10 and $20 \mathrm{~min}$ were necessary to achieve a steady state condition with a homogeneous dispersion. The steady state condition was assumed when density, pressure drop, droplet sizes, and temperature reached stable values. Visual inspection and the use of the impedance probes were also important to identify the flow pattern established in the acrylic pipe. When the steady state condition was attained measurements were performed starting from the lowest mixture velocity.

Pressure drop, flow rates, temperature, droplet sizes, and the impedance signals were obtained for a range of mixture velocities. With the measurements completed, the $90 \mathrm{~mm}$ pipe was changed by the 60 and then by the $32 \mathrm{~mm}$ pipe following the same procedure. To start the measurements at a new water cut the $90 \mathrm{~mm}$ pipe was placed back in the loop again. For the next water cut a volume of the oil-water mixture was removed keeping the loop running to guarantee that the removed mixture is homogeneous. Then, an equal volume of salt water was added to achieve the new water cut. The same procedure was followed for all the water cuts. A traversing gamma densitometer was used to study the dispersed phase distribution in the cross section of the $60 \mathrm{~mm}$ pipe. Measurements were performed with this instrument at each water cut at the mixture velocity of $1.0 \mathrm{~m} \cdot \mathrm{s}^{-1}$. The experimental procedure for the $16 \mathrm{~mm}$ pipe in the small flow loop was the same. Experiments started with single phase oil and salt water was added to get the different water cut conditions. The mixture velocities were also set starting from the lowest value and increasing it until the whole range was covered. All the experiments were performed at room temperature that was measured to be $23 \pm 2{ }^{\circ} \mathrm{C}$. Droplet size measurements were used to verify the flow development along the pipe. The FBRM probe was placed in two different positions in the $90 \mathrm{~mm}$ pipe (at $10 \mathrm{~m}$ and close to the outlet) to verify the droplet size variations. The $90 \mathrm{~mm}$ pipe was used for this test because of its smaller diameter-length ratio of all the pipes. It was observed that the droplet size distribution between these two points was the same. This leads to the conclusion that the droplets have reached a stable size and do not change in the last section of the acrylic pipe.

The experimental conditions tested in the present work are summarized in Table 2.

\section{Experimental results}

\subsection{Emulsion formation}

A digital microscope (Leica DM4000B) was used to see the structure and type of emulsion flowing in the pipe. Small samples were taken and visualized under the microscope at an intermediate velocity in the four pipe diameters at all water cuts. After the steady state condition was reached, it was observed that before inversion a stable water-in-oil dispersion was formed. This implies that all the added water was well dispersed by the oil continuous phase. Figure 3 shows a typical water-in-oil emulsion obtained before 
Table 2 Experimental matrix

\begin{tabular}{|c|c|c|c|c|}
\hline \multirow{2}{*}{ Water cut (\%) } & \multicolumn{4}{|c|}{ Velocity range $\left(\mathrm{m} \cdot \mathrm{s}^{-1}\right)$} \\
\hline & $16 \mathrm{~mm}$ ID & $32 \mathrm{~mm}$ ID & $60 \mathrm{~mm}$ ID & $90 \mathrm{~mm}$ ID \\
\hline $0,9,19,29,39,49$ & $\begin{array}{l}0.7,0.8,0.9,1.0,1.1,1.2,1.4,1.6 \\
1.8,2.0,2.2,2.4\end{array}$ & \multirow{7}{*}{$\begin{array}{l}0.5,0.6,0.7,0.8,0.9,1.0,1.2,1.4, \\
1.6,1.8,2.0,2.2,2.4,2.5,2.6,2.8 \\
3.0,3.2,3.4,3.5\end{array}$} & \multirow{7}{*}{$\begin{array}{l}0.5,0.6,0.7,0.8,0.9,1.0,1.2 \\
1.4,1.6,1.8,2.0,2.2,2.5\end{array}$} & \multirow{7}{*}{$\begin{array}{l}0.4,0.5,0.6,0.7,0.8,0.9, \\
1.0,1.1,1.2\end{array}$} \\
\hline 61 & $0.7-2.2^{\star}$ & & & \\
\hline 69 & $0.7-1.8^{*}$ & & & \\
\hline 76 & $0.7-1.4^{*}$ & & & \\
\hline 83 & $0.7-1.2^{*}$ & & & \\
\hline 90 & $0.7-1.1^{*}$ & & & \\
\hline 94 & $0.7-1.1^{*}$ & & & \\
\hline
\end{tabular}

${ }^{\star}$ Higher velocities were not reached due to a maximum operational pressure limitation of the loop.

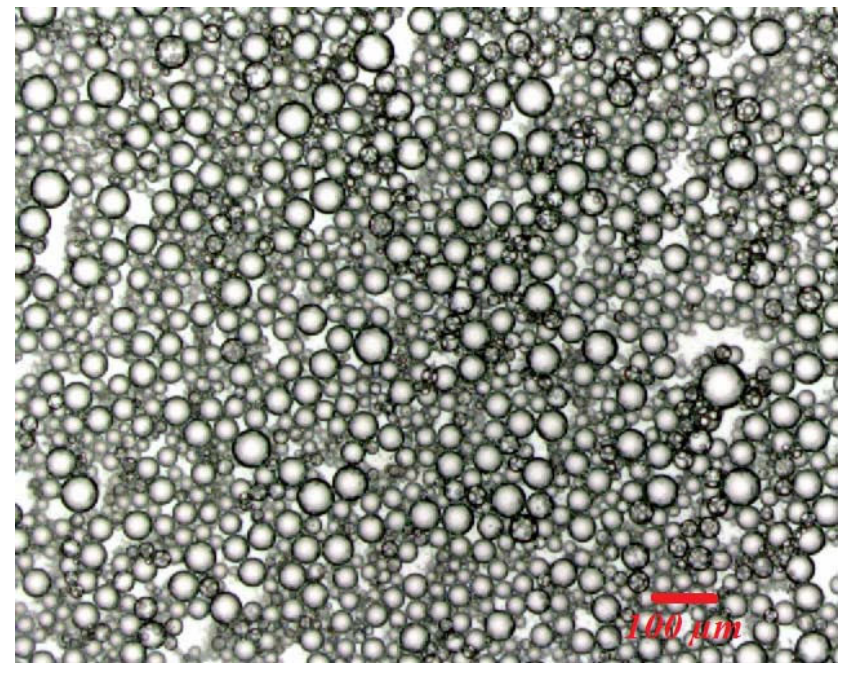

Fig. 3 Typical water-in-oil emulsion obtained before inversion in the pipe flow experiments (29\% water cut).

inversion sampled during the pipe flow. Although the pure oil and water phase were transparent, the formed emulsion looked white to the naked eye due to the presence of droplets. In addition to the small samples taken for the microscope visualizations, a one liter transparent recipient was used to verify the existence of separated water. Thus, when the steady state condition was reached the transparent recipient was used to take one liter samples from the four tested pipe diameters. This sampling was taken from the pipe outlet and at the lowest tested mixture velocities where a higher risk of water separation exists. These samples were kept in a quiescent condition for about one minute to verify the presence of separated water in the bottom of the recipient. Previous experiences with similar fluids showed that free water easily coalesces and sediments at quiescent conditions. No separated water was observed for the flow conditions before the occurrence of inversion confirming that water was well dispersed by the oil. The used samples were put back into the tank after the test.

\subsection{Dispersed phase distribution}

Water phase distribution in the pipe cross section was measured using a gamma densitometer. The main purpose of these measurements was to study the distribution of the dispersed phase in the pipe cross section rather than close to the walls where this instrument is less accurate. Measurements were performed only in the $60 \mathrm{~mm}$ pipe at the mixture velocity of $1.0 \mathrm{~m} \cdot \mathrm{s}^{-1}$. The dispersed phase was observed to be well distributed as shown in Fig. 4 .

\subsection{Pressure drop}

Pressure drop measurements as a function of water cut for different mixture velocities were obtained for the four pipe diameters. Figure 5 shows these results for (a) 90, (b) 60 , (c) 32, and (d) $16 \mathrm{~mm}$ IDs. The mixture velocities indicated in the figures correspond to the averaged reference values and may deviate $\pm 0.02 \mathrm{~m} \cdot \mathrm{s}^{-1}$ from the actual measured values.

As observed in Fig. 5 that pressure drop increases as the mixture velocity gets higher for constant water cuts. However, a peculiar behavior is observed for constant mixture velocities as a function of water cut.

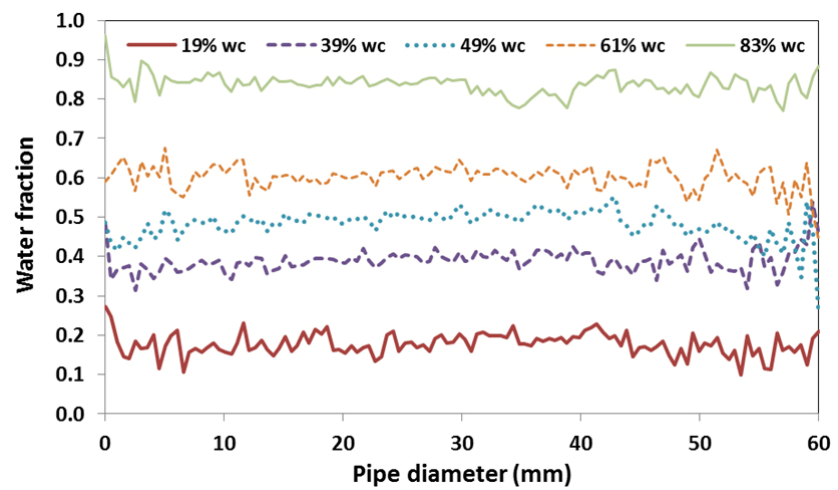

Fig. 4 Water fraction measurements using the gamma densitometer. 

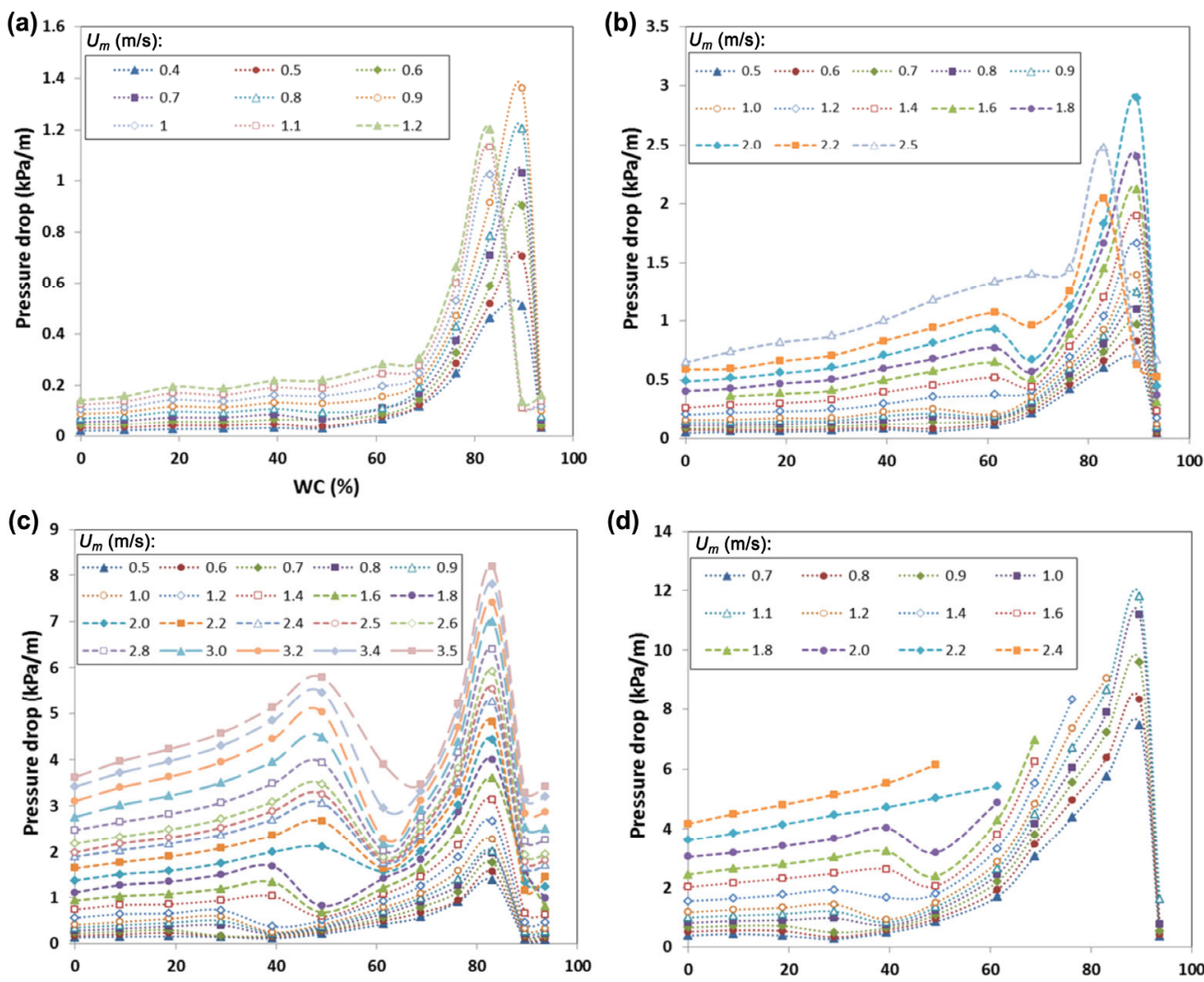

Fig. 5 Pressure drop as a function of water volume fraction for pipes for varying mixture velocities: (a) $D=90 \mathrm{~mm}$, (b) $D=60 \mathrm{~mm}$, (c) $D=32 \mathrm{~mm}$, (d) $D=16 \mathrm{~mm}$.

In the $90 \mathrm{~mm}$ pipe pressure drop slightly increases with water cut between 0 and $61 \%$ for a constant mixture velocity. A steeper increase is observed from $69 \%$ to $90 \%$ water cut that is finally reduced due to the occurrence of phase inversion. A similar trend is observed for the $60 \mathrm{~mm}$ pipe. However, in this case pressure drop slightly reduces at $69 \%$ water cut for the mixture velocities ranging from 1.4 to $2.2 \mathrm{~m} \cdot \mathrm{s}^{-1}$. The same occurs for the mixture velocities from 0.8 to $1.0 \mathrm{~m} \cdot \mathrm{s}^{-1}$ at $61 \%$ water cut. This behavior is more evident in the $32 \mathrm{~mm}$ pipe (Fig. 5(c)) where a wider range of mixture velocities was tested. For the $32 \mathrm{~mm}$ pipe this reduction of pressure drop is present at about $29 \%$ for $0.5-0.7 \mathrm{~m} \cdot \mathrm{s}^{-1}, 39 \%$ for $0.8-1.2 \mathrm{~m} \cdot \mathrm{s}^{-1}, 49 \%$ for $1.4-1.8 \mathrm{~m} \cdot \mathrm{s}^{-1}$, and $61 \%$ water cut for the mixture velocities from 2.0 to $3.5 \mathrm{~m} \cdot \mathrm{s}^{-1}$. Figure $5(\mathrm{~d})$ shows the pressure drop results obtained from the $16 \mathrm{~mm}$ pipe. Similar trends are seen in this pipe diameter as water cut increases for a constant mixture velocity. This time less data points were measured at high water cuts where the emulsion viscosity increases considerably. This was a result of a maximum operational pressure limitation in the smaller flow loop which prevented of acquiring data at the highest mixture velocities.

Pressure drop measurements as a function of the mixture velocity presented in Fig. 6(a) shows these results for the $32 \mathrm{~mm}$ pipe while Fig. 6(b) presents the same results for the $90 \mathrm{~mm}$ pipe at the indicated water cuts. Together with the measurement equations (45) and (46) are also plotted. These two equations are used as a tool to identify the flow condition in the pipe by tuning the factors in parenthesis with the experimental data. It is observed in Fig. 6 that for the $32 \mathrm{~mm}$ pipe at $19 \%$ water cut the measurements follow very well the turbulent behavior equation (46). As the water cut is increased to $39 \%$ the flow condition becomes laminar at mixture velocities lower than $1.0 \mathrm{~m} \cdot \mathrm{s}^{-1}$. At velocities higher than $1.4 \mathrm{~m} \cdot \mathrm{s}^{-1}$ for the same water cut turbulent flow conditions remain. At $61 \%$ water cut most of the tested conditions were laminar except for the highest mixture velocities (3.4 and $3.5 \mathrm{~m} \cdot \mathrm{s}^{-1}$ ) that appear to be in the transition region (not turbulent either). On the other hand, Fig. 6(b) shows that for the $90 \mathrm{~mm}$ pipe the initial signs of laminar flow are present at $49 \%$ water cut. Finally, at $69 \%$ water cut the flow is laminar for the whole range of tested velocities.

The results shown in Fig. 6 indicate that for a constant mixture velocity, a turbulent-laminar transition occurs as the water cut increases (due to the higher effective viscosities). This is in agreement with the trends observed in Fig. 5 were pressure drop decreases at some conditions. For instance, the results at $49 \%$ water cut in Fig. 6(a) show that transition occurs between 1.8 and $2.0 \mathrm{~m} \cdot \mathrm{s}^{-1}$ which agrees with results 

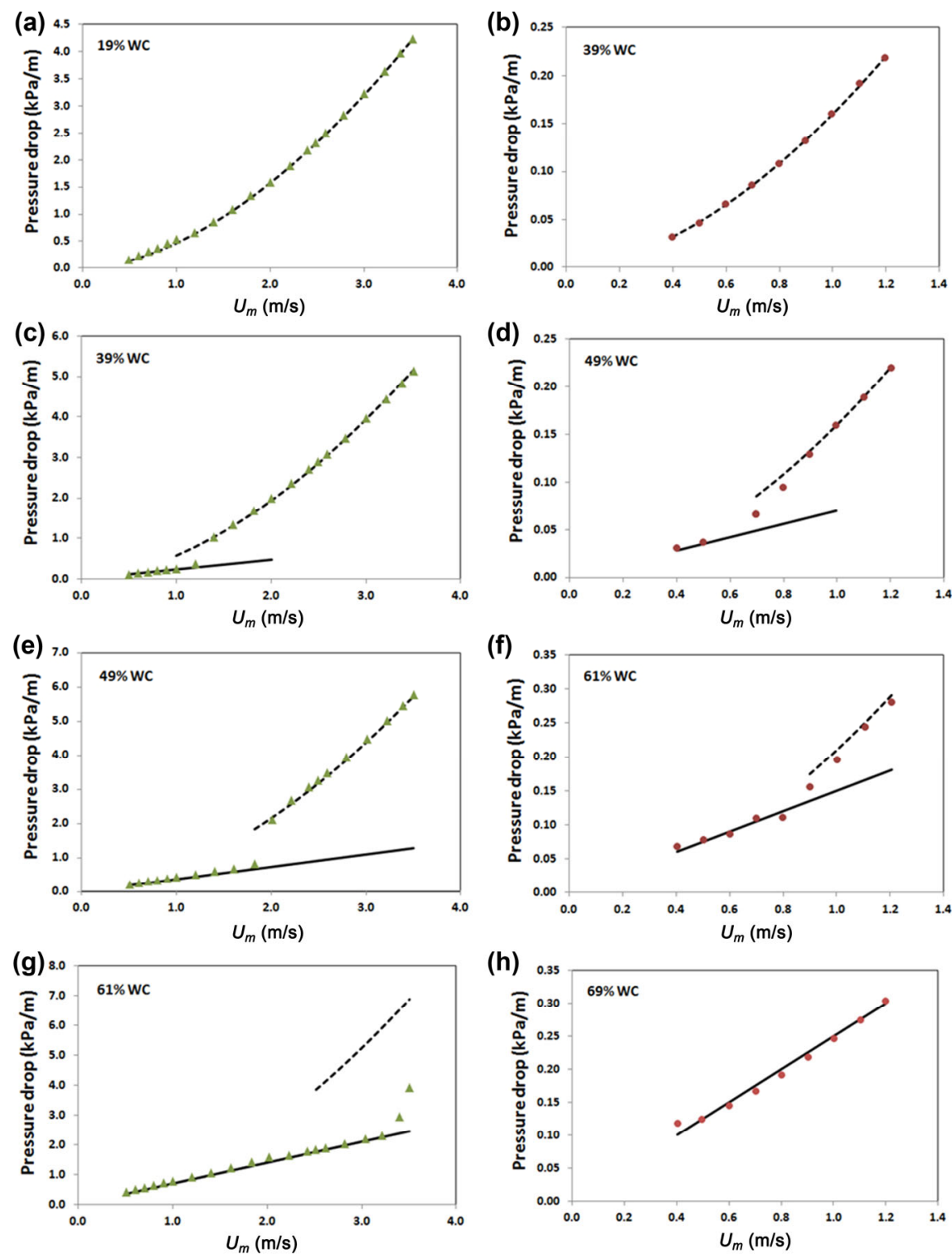

Fig. 6 Pressure drop as a function of the mixture velocity for (a) $32 \mathrm{~mm}$ (green) and (b) $90 \mathrm{~mm}$ (red) pipe. Turbulent (dashed) and laminar (solid) flow.

observed in Fig. 5(c). This turbulent-laminar transition was observed in all pipe diameters where at low water cuts turbulent conditions were established and above $61 \%$ water cut the flow conditions were all laminar. For all the tested conditions turbulent flow became laminar at low or medium water fractions in the four pipe diameters. A turbulent condition was not possible close to the inversion point. It might be interesting to study its effect on the occurrence of phase inversion. As observed in Fig. 5(c), the pipe flow of the water-in-oil emulsion shows a higher pressure drop in turbulent conditions similar to single phase flow. The pressure drop reduces when the flow gets into the transition and finally in the laminar condition.

The phase inversion phenomenon in pipe flow conditions can also be identified as a dramatic reduction of pressure drop. The pressure drop reduction due to the turbulentlaminar transition observed in Fig. 5 could be wrongly interpreted as the occurrence of phase inversion if it is not carefully analyzed. In the present work, phase inversion was observed to occur at a very high water cut for the tested conditions. It was identified by visual observation, pressure drop measurements and with the use of conductance probes. Up to $90 \%$ water cut the mixture was still oil continuous (water-in-oil emulsion) at relatively low flow rates. As the mixture velocity was increased the inversion process was triggered and a dispersion of water-in-oil in water was established accompanied by an abrupt reduction of pressure drop. This is observed in Fig. 5(a), for the $90 \mathrm{~mm}$ pipe, where the pressure reduces substantially when increasing the mixture velocity from 0.9 to $1.0 \mathrm{~m} \cdot \mathrm{s}^{-1}$. This inversion 
process occurred in a similar way for the $60 \mathrm{~mm}$ pipe. Figure 5(b) shows a large difference in pressure drop between the mixture velocities of 2.0 and $2.2 \mathrm{~m} \cdot \mathrm{s}^{-1}$ which clearly indicates that inversion took place as the mixture velocity increased. For the $16 \mathrm{~mm}$ pipe (Fig. 5(d)) oil was still continuous at $90 \%$ water cut for the tested mixture velocities. In the case of the $32 \mathrm{~mm}$ pipe (Fig. 5(c)) water-in-oil was not possible at $90 \%$ water cut which is confirmed by the pressure drop measurements. The occurrence of phase inversion in these experiments will be further discussed in a next section. The reasons for such differences will be commented upon.

\subsection{Pressure drop uncertainties}

The uncertainties related to pressure drop measurements are presented in Fig. 7. The results are shown for the $32 \mathrm{~mm}$ ID pipe for selected mixture velocities.

The plotted uncertainties are estimated as two times the standard deviation of the measurements. In general, the uncertainties are small and show a small increase as flow rate increases. Also, for constant mixture velocities, the uncertainties appear to be slightly higher as the flow gets into the transition region. The indicated mixture velocities associated to the pressure drop curves are nominal. Small deviations from the indicated mixture velocities were inevitable despite of the effort to avoid them. This is due to fine tuning limitations when trying to set the frequency in the variable speed drivers that were controlling the pumps.

The actual mixture velocities are presented for reference in Fig. 8. Another possible source of uncertainty was related to the density of the emulsion mixture. The oil-water mixture was in general stable and homogeneous, which was verified by sampling and density measurements in the Coriolis flow meter.

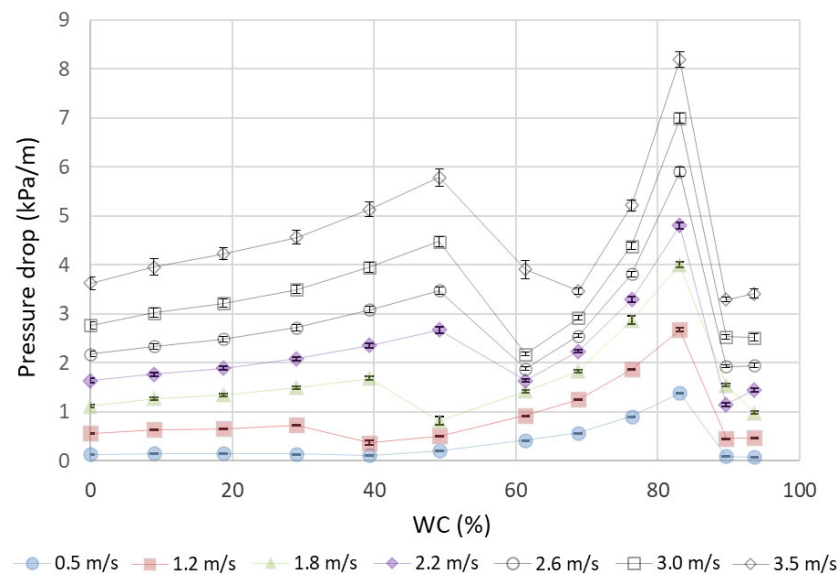

Fig. 7 Pressure drop measurements with their associated uncertainties for different mixture velocities in the $32 \mathrm{~mm}$ ID pipe $(\times 2$ standard deviation).

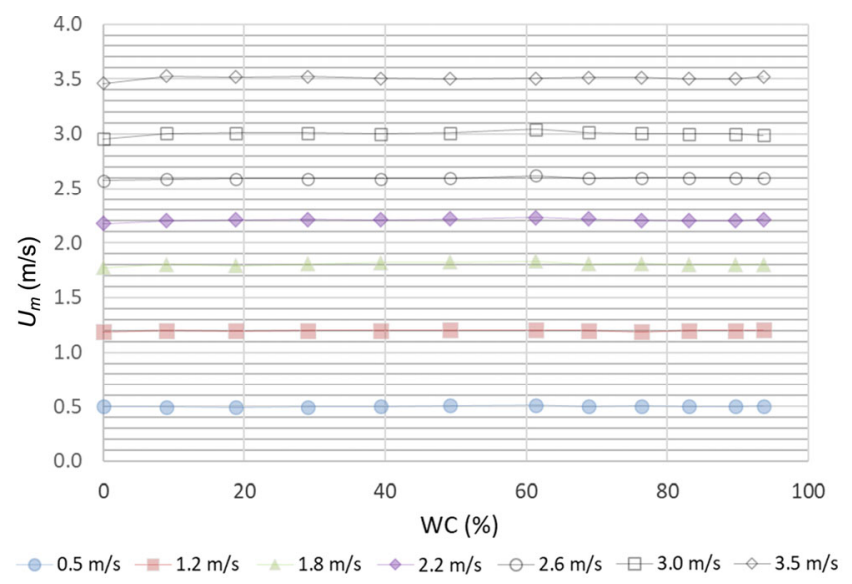

Fig. 8 Actual mixture velocities for each of the pressure drop measurements.

\subsection{Drag reduction effect}

A drag reduction effect in pipe flow of dispersed liquid-liquid flows has been reported by some authors (Pal, 1993, 2007; Angeli and Hewitt, 1999; Ioannou et al., 2005; Plasencia and Nydal, 2010). This drag reduction is attributed to the effect that droplet breakup and coalescence have on turbulence (Pal, 1993). Results show that this effect is common in unstable oil-water dispersed flows where breakup and coalescence is frequent. In the present study, no drag reduction effect was observed for the tested conditions. Figure 9 presents the friction factors calculated from
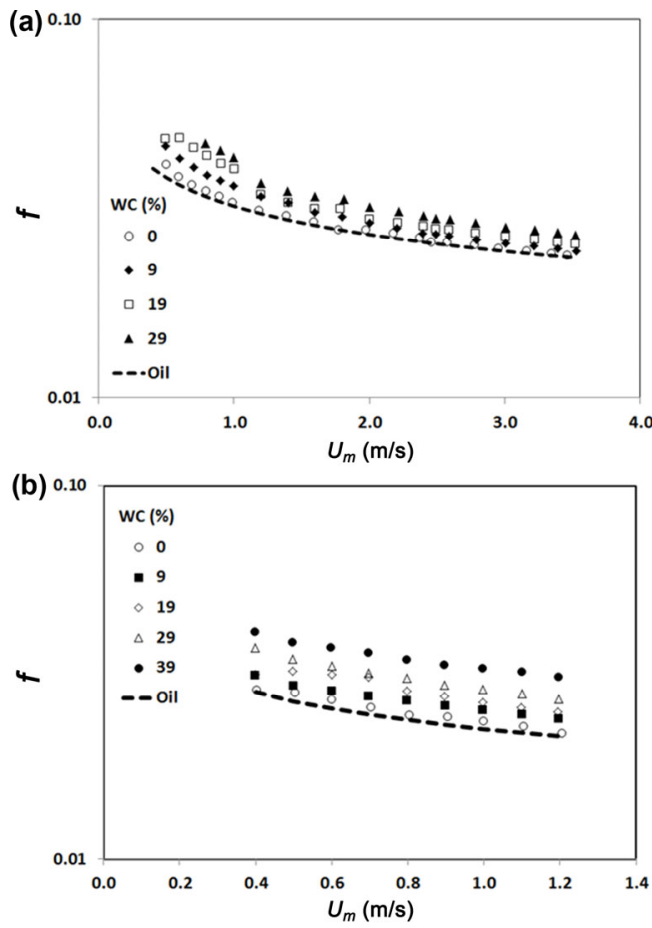

Fig. 9 Friction factors as a function of the mixture velocity at different water cuts for turbulent flow conditions: (a) $32 \mathrm{~mm} \mathrm{ID}$, (b) $90 \mathrm{~mm}$ ID. 
pressure drop measurements using Eq. (41) for the pipe diameters of 32 and $90 \mathrm{~mm}$. Only the data points that correspond to turbulent flow conditions are included here. Results presented in Fig. 9 show that friction factors increase with water cut in both pipe diameters. In Fig. 9, the dashed lines indicate the theoretical values calculated for single phase oil.

\subsection{Phase inversion}

The phase inversion phenomenon in pipe flow has been mainly reported to be a result of an increase of the dispersed phase volume fraction (Cengel et al., 1962; Arirachakaran et al., 1989; Nädler and Mewes, 1997; Angeli and Hewitt, 1999; Lovick and Angeli, 2004; Ioannou et al., 2005; Piela et al., 2006; Plasencia and Nydal, 2010). The wettability effect of the fluids on the pipe wall has an influence on the occurrence of phase inversion (Angeli and Hewitt, 1999; Ioannou et al., 2005). Hence, the phase that better wets the pipe wall will remain as the continuous phase for a wider range of phase fractions. Also, pre-wetting the pipe wall with one or the other phase will affect the occurrence of phase inversion. In the present work, the experiments were started with single phase oil and the aqueous phase was increased in steps. The inverse process (starting with single water phase and increasing oil) was not performed.

The inversion process occurred at a very high water cut (90\%) and was triggered by an increase of the mixture velocity (shear rate). Thus, two different types of emulsions, a water-in-oil emulsion and a water-in-oil in water emulsion, were possible at the same water cut. Hence, the initial water-in-oil emulsion inverted into a water-in-oil in water multiple emulsion by just increasing the mixture velocity. This occurred at 1.0 and $2.2 \mathrm{~m} \cdot \mathrm{s}^{-1}$ in the 90 and $60 \mathrm{~mm}$ pipes respectively. Figure 10 shows pictures of (a) water-in-oil emulsion and (b) water-in-oil in water multiple emulsions obtained before and after inversion respectively. Both pictures were taken at $90 \%$ water cut before and right after inversion. The fluid in Fig. 10(a) presents a white homogeneous color while white droplets flow in a transparent continuous phase (salt water) in Fig. 10(b). These white droplets are in fact a dispersed water-in-oil emulsion. The water-in-oil emulsions obtained prior to the occurrence of inversion resulted to be stable. In contrast, the formation of the multiple emulsions (water continuous) after inversion was unstable and lead to segregation of the dispersed emulsion droplets and water under quiescent conditions.

Pressure drop as a function of the mixture velocity for 83\%, 90\%, and 94\% water cut are shown in Fig. 11 for the $90 \mathrm{~mm}$ pipe. It is possible to see the pressure drop increasing with the mixture velocity at $83 \%$ water cut where the fluid was still a water-in-oil emulsion. At $90 \%$ water cut pressure

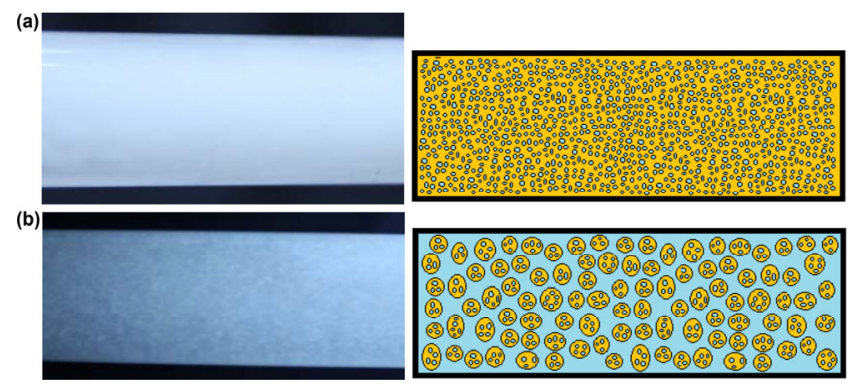

Fig. 10 (a) Water-in-oil emulsion (w/o) and (b) water-in-oil in water $(\mathrm{w} / \mathrm{o} / \mathrm{w})$ multiple emulsion obtained in the experiments.

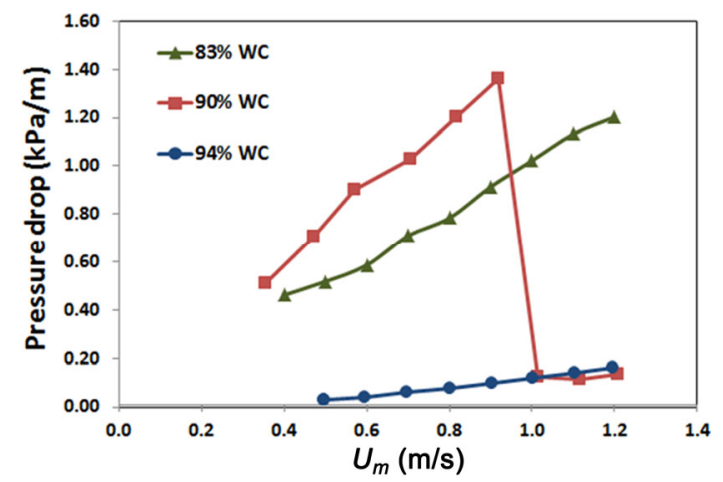

Fig. 11 Pressure drop as a function of mixture velocity at different water cuts in the $90 \mathrm{~mm}$ pipe.

drop dramatically decreases from 0.9 to $1.0 \mathrm{~m} \cdot \mathrm{s}^{-1}$ showing evidence of a flow structure change from oil continuous to water continuous as presented in Fig. 10. Finally, at $94 \%$ water cut, pressure drop continues to be low due to the presence of water as the continuous phase. The same effect was observed in the $60 \mathrm{~mm}$ pipe when increasing the mixture velocity from 2.0 to $2.2 \mathrm{~m} \cdot \mathrm{s}^{-1}$.

The shear induced inversion process observed in the experiments resulted to be reversible. In the same way that the inversion occurred by increasing the mixture velocity, the multiple emulsion inverted back into a water-in-oil emulsion just by reducing the mixture velocity. Nevertheless, this process presented hysteresis.

As the mixture velocity was reduced, the multiple emulsions suffered a sequence of flow pattern transformations as presented in Fig. 12. The initially well dispersed water-in-oil droplets flowing in water (Fig. 12(a)) started to concentrate in the upper portion of the pipe as observed in Fig. 12(b). A coalescence process then started forming (c) a layer of large bubbles of water-in-oil emulsion flowing in the continuous water phase close to the top of the pipe. At this point the oil phase did not seem to be in contact with the pipe wall. Simultaneously to the coalescence process, free water was dispersed back into the oil phase resulting in larger volumes of the water-in-oil emulsion (dispersed phase increasing) which almost occupies the whole section of the pipe (d). At this stage a combination of annular and 


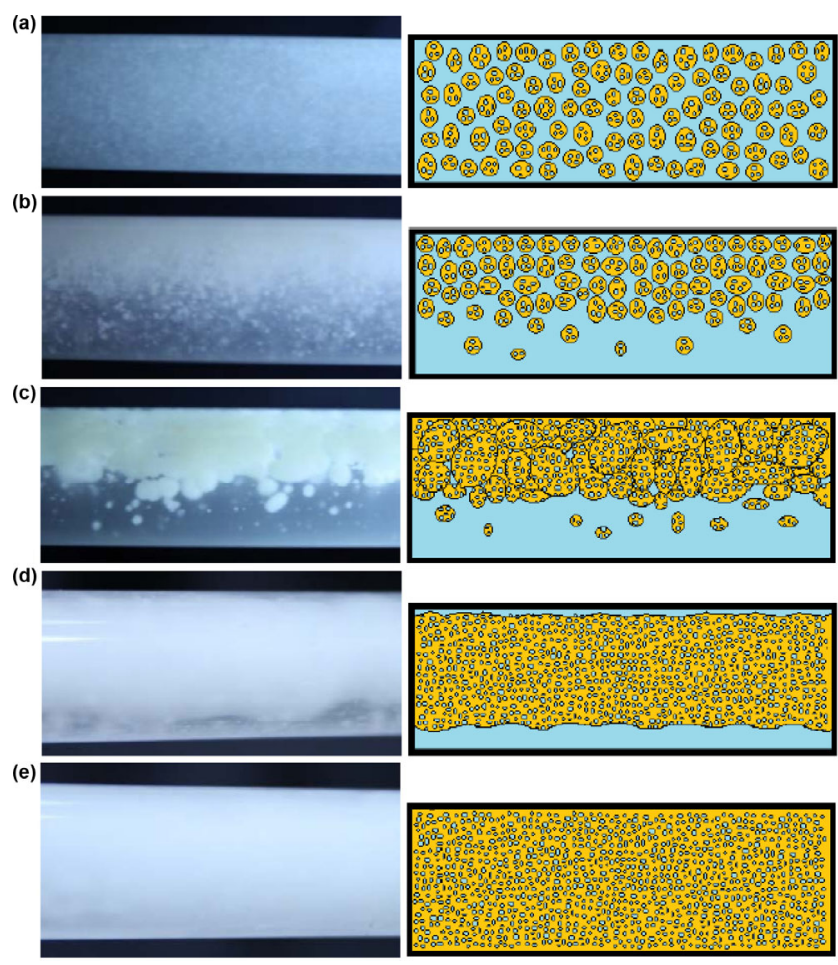

Fig. 12 Flow patterns observed during the inversion process from (a) water-in-oil in water emulsion $(w / o / w)$ to (e) water-in-oil emulsion (w/o).

stratified like flows was observed were the water-in-oil emulsion was the core surrounded by water. The annular like flow pattern easily switches to a stratified like flow pattern when the oil phase gets in contact with the pipe wall. For the water continuous flow (after inversion) the annular regime was more frequent in the smaller pipe diameters (16 and $32 \mathrm{~mm}$ pipes) while a stratified like flow pattern tend to dominate in the larger pipe diameters (60 and $90 \mathrm{~mm}$ pipes). As the mixture velocity was further reduced the water-in-oil emulsion was able to disperse all the surrounding water reaching the pipe wall and suddenly increasing the pressure drop. This process took approximately $20 \mathrm{~min}$ period in which the velocity was gradually reduced from 2 to $0.5 \mathrm{~m} \cdot \mathrm{s}^{-1}$ in the $60 \mathrm{~mm}$ pipe and from 1.2 to $0.6 \mathrm{~m} \cdot \mathrm{s}^{-1}$ in the $90 \mathrm{~mm}$ pipe. The flow regimes presented in Fig. 12 are most likely similar to the ones that crude oil may present after inversion when no chemical additives are present. It has to be considered that the water-in-oil emulsion flowing in the water continuous phase disperses more water when going from Figs. 12(a) to 12(e). This results in higher effective viscosities of the flowing water-in-oil emulsion. Then, at least three parameters are affecting the flow regimes presented here. One is the mixture velocity, also the amount of separated water and the finally the effective viscosity of the water-in-oil emulsion which is affected by the local dispersed water fraction.
The flow patterns (c) and (d) presented in Fig. 12 have similarities with the ones obtained in highly viscous oil-water flow experiments (Bannwart et al., 2004). The formation of an annular like flow pattern and a bubble regime has also been reported to be present in highly viscous oil-water flow (Bannwart et al., 2004). This is a result of the higher viscosity of the water-in-oil emulsion flowing in the water continuous phase. The shear induced inversion was observed at $90 \%$ water cut in the 90 and $60 \mathrm{~mm}$ pipes. In the $32 \mathrm{~mm}$ pipe the $90 \%$ water-in-oil emulsion was not completely achieved which is confirmed by the low pressure drops (Fig. 5). With the $32 \mathrm{~mm}$ pipe connected to the loop the fluids were circulated looking for the steady state water-in-oil emulsion obtained at low velocities in the 60 and $90 \mathrm{~mm}$ pipe. Here, as the fluids were circulated, pipe sections with a water-in-oil emulsion and others with separated water were obtained. It was observed that the water-in-oil emulsion sections were getting larger with time. Although the system was going towards the establishment of a water-in-oil emulsion (as in the larger pipes), a stable condition was reached without completing the process. This is attributed to a small accumulation of oil in the tank which in fact results in higher local water cuts in the pipe. At this very high water cut (90\%) it is much easier for water and oil to segregate. In the larger pipes the water-in-oil emulsion was obtained at relatively low mixture velocities that in the case of the $32 \mathrm{~mm}$ pipe resulted in very small flow rates. It was observed that in the $32 \mathrm{~mm}$ pipe at $90 \%$ water cut the mixture density measurements were proportional to the water cut for mixture velocities above $1.5 \mathrm{~m} \cdot \mathrm{s}^{-1}$ (which resulted in a water-in-oil in water flow regime Figs. 12(a) and 12(b)). However, the mixture density slightly increased for lower mixture velocities giving rise to a virtually higher water cut (oil accumulation in the tank). The $32 \mathrm{~mm}$ pipe was connected to the large flow loop where all the loop pipe diameters are considerably larger which was appropriate at lower water cuts but it is not for this very high water cut (90\%). This leads to longer residence time which is critical for such a high water cut. Then, a small accumulation of oil in the tank prevented of obtained the water-in-oil emulsion results than in the larger pipes due to higher local water cuts.

The results in the $16 \mathrm{~mm}$ pipe show that at $90 \%$ water cut an emulsion of water-in-oil is also possible in small pipes. This indicates that the results in the $32 \mathrm{~mm}$ pipe were not due to a diameter effect but it occurred as a result of oil accumulation in the tank. The $16 \mathrm{~mm}$ pipe diameter was connected to a separated small scale loop where oil or water accumulation in the tank was easily avoided. The formation of multiple emulsions (occurrence of inversion) was not observed at $90 \%$ water cut in the $16 \mathrm{~mm}$ pipe. 
Higher mixture velocities that may trigger the inversion process were not achieved due to a pressure limitation in the loop. The assessment performed on the shear-driven occurrence of inversion suggests that the flow loop physical restrictions played a key role. It is believed that phase inversion in the 60 and $90 \mathrm{~mm}$ pipes occurred in the Coriolis flow meter. The internal pipe diameter of the Coriolis flow meter is $35 \mathrm{~mm}$ (see Fig. 2). The inversion process occurred at 1.2 and $2.2 \mathrm{~m} \cdot \mathrm{s}^{-1}$ in the 90 and $60 \mathrm{~mm}$ pipes which corresponds to a flow rate of about $0.006 \mathrm{~m}^{3} / \mathrm{s}$ in both pipes. At $90 \%$ water cut a mixture velocity of the order of $6.6 \mathrm{~m} \cdot \mathrm{s}^{-1}\left(0.006 \mathrm{~m}^{3} / \mathrm{s}, 35 \mathrm{~mm}\right.$ ID) was necessary to invert the dispersion. Thus, the droplets are forced to be smaller (tendency to be mono-disperse) which triggers phase inversion due to high water cut (90\% higher that maximum packing number for mono-disperse systems). Mixture velocities in the smaller pipe diameters were by far lower than $6.6 \mathrm{~m} \cdot \mathrm{s}^{-1}$ which prevented the occurrence of the same mechanism.

\subsection{Droplet size}

Typical in-situ chord length distributions acquired with the FBRM probe before inversion are shown in Fig. 13 at different water cuts. This shows a tendency of droplets sizes to grow as the concentration of the dispersed phase increases in large and small pipes. The effect of the mixture velocity on the droplet sizes for a constant water fraction is illustrated in Fig. 14 for 32 and $90 \mathrm{~mm}$ pipes. It is observed that droplet sizes get smaller as the mixture velocity increases in both pipe diameters. A smaller variation occurs in the $90 \mathrm{~mm}$ pipe where a narrow velocity range was tested when compared with the $32 \mathrm{~mm}$ pipe where velocities up to $3.5 \mathrm{~m} \cdot \mathrm{s}^{-1}$ were possible.

The inversion process involved a change in the droplet size distribution. Droplet sizes for the 60 and $90 \mathrm{~mm}$ pipes before and after inversion are presented in Fig. 15. Larger droplet sizes are observed before inversion (up to $0.9 \mathrm{~m} \cdot \mathrm{s}^{-1}$ in $90 \mathrm{~mm}$ and $2.0 \mathrm{~m} \cdot \mathrm{s}^{-1}$ in $60 \mathrm{~mm}$ ID) and they suddenly get smaller when increasing the mixture velocity. This might indicate that the larger droplets coalesced to form the continuous phase and only small ones remain dispersed in the water-in-oil droplets that are the new dispersed phase. However, the FBRM technique used to study droplet sizes measures the chord length that in the case of multiple
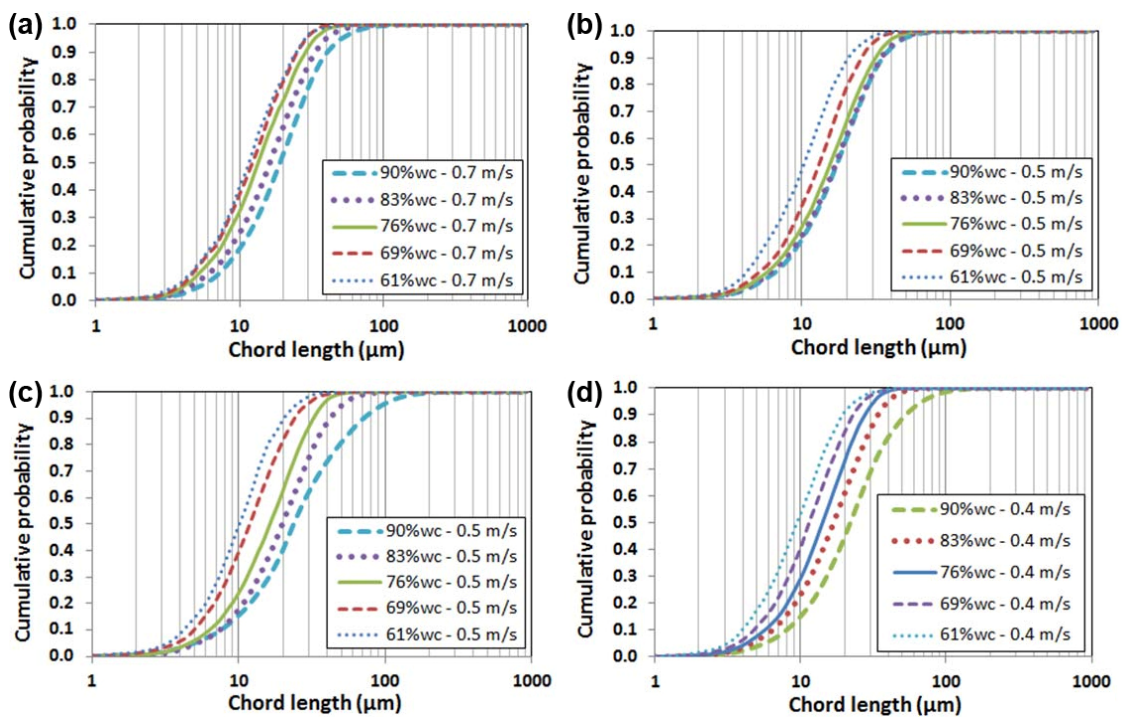

Fig. 13 Chord length measurements at different water cuts: (a) $16 \mathrm{~mm}$ pipe at $0.7 \mathrm{~m} \cdot \mathrm{s}^{-1}$, (b) $32 \mathrm{~mm}$ pipe at $0.5 \mathrm{~m} \cdot \mathrm{s}^{-1}$, (a) $60 \mathrm{~mm}$ pipe at $0.5 \mathrm{~m} \cdot \mathrm{s}^{-1}$, and (d) $90 \mathrm{~mm}$ pipe at $0.4 \mathrm{~m} \cdot \mathrm{s}^{-1}$.
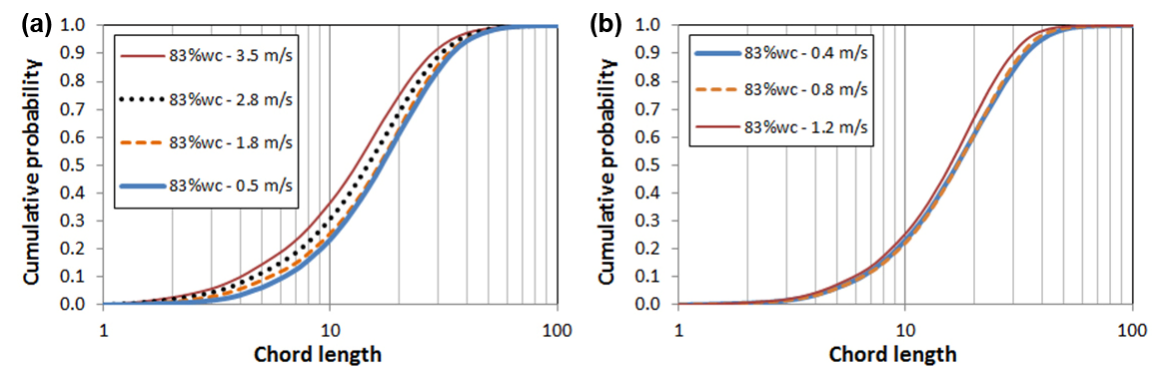

Fig. 14 Chord length distributions at different mixture velocities at constant water cuts: (a) $32 \mathrm{~mm}$, (b) $90 \mathrm{~mm}$. 

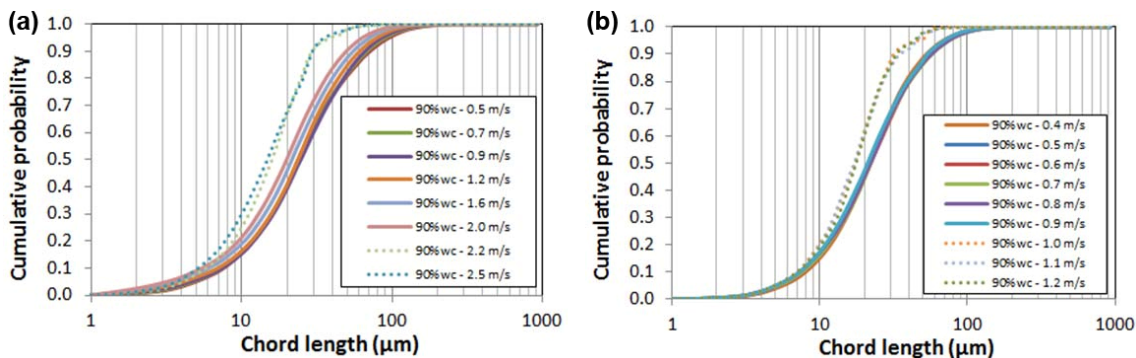

Fig. 15 Droplet size distributions at 83\% water cut: (a) $60 \mathrm{~mm}$ and (b) $90 \mathrm{~mm}$ ID.

emulsions (after inversion) may lead to results that are of difficult interpretation.

\subsection{Presence of water on the pipe wall}

Conductance probes were also used to detect the presence of water at the pipe wall. Conductance probes were used for the 32,60, and $90 \mathrm{~mm}$ pipes and were not available for the $16 \mathrm{~mm}$ pipe. They were located at two positions, in the middle and close to the outlet of the acrylic pipe. A special calibration test had to be made due to the use of salt water in the experiments. The addition of salt in the water phase increases its electrical conductivity which saturates the signal in the presence of small amounts of salt water in contact with the wall. A $1 \mathrm{~mL}$ syringe was used to measure the amount of salt water needed to saturate the signal. Using the syringe, the salt water was homogeneously distributed as a film between the two rings. Volumes of 0.5 and $1.2 \mathrm{~mL}$ were necessary to saturate the signal in the 32 and $90 \mathrm{~mm}$ pipes. A larger volume in the $90 \mathrm{~mm}$ pipe was necessary because of the longer distance between the rings in the large pipe. With the volume of salt water and the distance between the rings, it was calculated that a liquid film with a transversal area of approximately $30 \mathrm{~mm}^{2}$ is necessary to saturate the signal at $10.5 \mathrm{~V}$. Above this small liquid film of water the probes will saturate. For this reason the conductance probes were used to detect whether there is or not water in contact with the pipe wall and not to quantify the local amount.

Figure 16 shows typical signals at different water cuts obtained for the $60 \mathrm{~mm}$ pipe at $1.4 \mathrm{~m} \cdot \mathrm{s}^{-1}$. After inversion, at

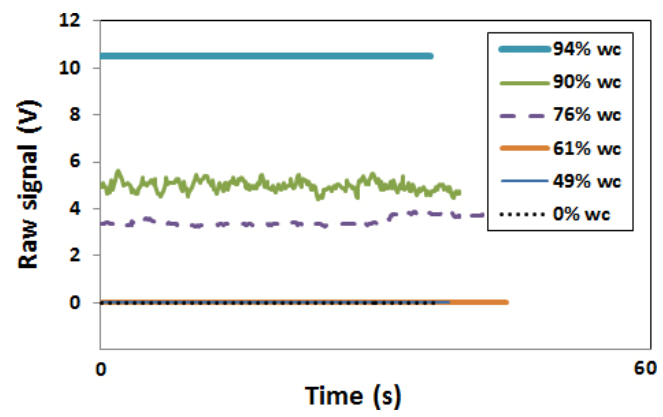

Fig. 16 Conductance probe signal as a function of water cut (pipe ID $60 \mathrm{~mm}, 1.4 \mathrm{~m} \cdot \mathrm{s}^{-1}$ ).
94\% water cut, the presence of water as the continuous phase saturates the signal. The conductance probes were observed to detect the presence of water above $61 \%$ water cut. However, for the studied conditions the signal was always lower than $10.5 \mathrm{~V}$, typically $5-8 \mathrm{~V}$ at the higher water cuts before inversion (higher velocities showed lower signals). This indicates that no significant amounts of water were in contact with the pipe wall even at high water cuts before inversion. This is in agreement with the sampling tests taken in a transparent recipient where no appreciable separated water was observed.

\subsection{Presence of multiple emulsions}

Water-in-oil was the dominant type of emulsion before the occurrence of phase inversion, where oil was the continuous phase wetting the pipe wall. The presence of multiple emulsions always results in higher effective dispersed phase fractions. Then, a hypothetical oil-in-water in oil multiple emulsion would most probably trigger inversion at lower water cuts than $90 \%$ due to the higher effective dispersed phase fraction, which was not the case. Microscopic visualization of the samples taken at different water cuts did not show evidence of the formation of oil-in-water in oil multiple emulsions before inversion. Microscopic visualization at very high water cuts (typically $80 \%-90 \%$ ) is a challenge. Water droplets rapidly coalesced upon contact with the glass slide resulting in a multiple type of emulsion that might not be real in pipe conditions. After inversion, water-in-oil in water multiple emulsions were visualized under the microscope. Figure 17 shows a typical picture of such emulsions taken with the microscope.

\subsection{Flow loop pump influence on the emulsion formation}

The pumps may have an effect on the mixing and emulsions formation (droplet sizes). In addition, as observed in Fig. 2, the flow loop has some small diameter pipe sections which include the Coriolis flow meter (ID $35 \mathrm{~mm}$ ). They had to be assumed as limitations of the experimental set-up. The pump effect is expected to be critical at high flow rates were 


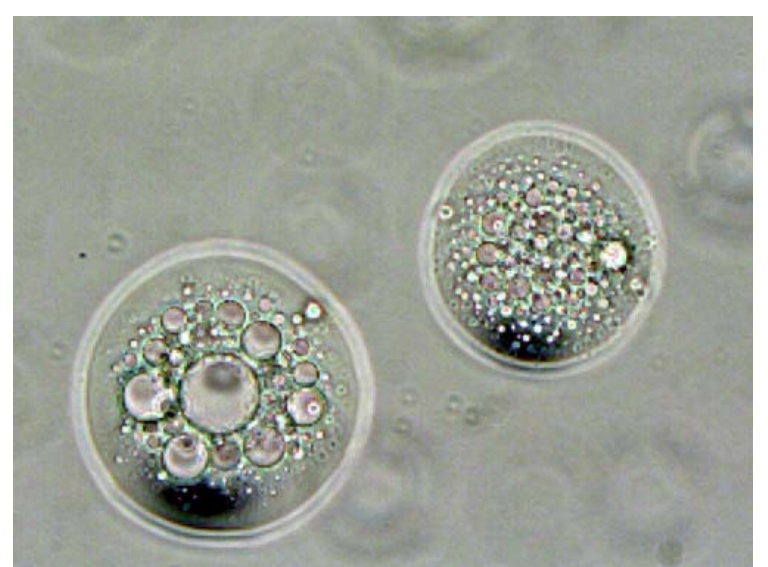

Fig. 17 Presence of multiple emulsions (water-in-oil in water) after the occurrence of inversion.

the rotor speed is higher (about $160 \mathrm{rpm}$ for the maximum flow rate used, $0.0076 \mathrm{~m}^{3} / \mathrm{s}$ ). The maximum flow rate was used in the 60 and $90 \mathrm{~mm}$ pipes while was not reached in the $32 \mathrm{~mm}$ pipe due to the lower flow rates (about $70 \mathrm{rpm}$, $0.0028 \mathrm{~m}^{3} / \mathrm{s}$ ). Looking at Fig. 14 it is observed that droplet sizes are very similar in the 32 and $90 \mathrm{~mm}$ at the lower mixture velocities $(60 \%$ of droplets smaller than $20 \mu \mathrm{m})$. Furthermore, even smaller droplets are observed in the $32 \mathrm{~mm}$ pipe when increasing the mixture velocity. A major effect of the pumps would result in smaller droplet sizes in the larger pipe diameters due to the higher flow rates used which is not the case. This supports the assumption that the pumps did not have a major effect on the droplet formation. A significant effect of the pump on the emulsion would most likely trigger inversion at lower water cuts than $90 \%$ by promoting coalescence making it difficult to reach such a high amount of dispersed water. It is believed that the inversion process presented here was driven by increasing the shear rate due to the reduced section of the Coriolis flow meter. This is concluded because the phase inversion was reproducible (occurred at the same mixture velocity) and was driven by a small increase in the mixture velocity.

\section{CFD analysis}

We have run a set of simulations using a development version of Siemens Simcenter3D STAR-CCM+. We have run axisymmetric simulations (neglecting gravity) and 3D simulations with gravity in a 3D half-pipe with a vertical plane of symmetry to reduce the cell count.

\subsection{Mesh}

Axisymmetric simulations made use of a grid with cells getting finer towards the wall in order to produce a low $y^{+}$for the turbulent simulations (see Section 2.3.2). As $y^{+}$is a function of velocity, phase density, and viscosity (Eq. (37)), it varies for the phases and the different velocities studied. For most of the simulations it lays in the range $1<y^{+}<20$ meaning the turbulence was well resolved with that mesh. The axisymmetric mesh has 30 cells across the radius of the pipe and 3500 cells along the axis giving a total of 105,000 cells.

For the $3 \mathrm{D}$ case we used a mesh of a half pipe with one plane of symmetry consisting of 375,000 cells (Fig. 18). The simulations were run on local workstations in double precision in parallel with transient flow and a time-step of $\mathrm{d} t=0.0001-0.01 \mathrm{~s}$ depending on their ability to converge to a solution (for the dense emulsions with high mixture viscosities the equations become stiff and required a smaller time-step). The simulations were run until steady state at $t=100 \mathrm{~s}$. Using the method outlined in Section 2.5.1, we identify which simulations are run as laminar or turbulent. All of the simulations except for the pure oil or pure water cases are modeled with the Eulerian multi-phase model outlined in Section 2.2.1 and used the modified Krieger and Dougherty model described in Section 2.1. Whilst data has been provided for the distribution of droplet sizes in Section 4.7, we have made the assumption that the droplets are of a constant mean diameter of $150 \mu \mathrm{m}$ as the modified Krieger and Dougherty model is independent of droplet size so therefore this quantity only enters the calculations in the form of the drag force acting between the phases (Section 2.2.2). We ran a set of axisymmetric simulations for $\phi=0.29$ and $U=1.8 \mathrm{~m} \cdot \mathrm{s}^{-1}$ with droplet sizes from $1 \times 10^{-5}$ to $1 \times 10^{-3} \mathrm{~m}$ and we confirmed we got the same pressure drop in all cases (results not shown). There is some experimental evidence that the size of the droplets (and therefore the size distribution) becomes important as the droplets approach maximum packing as $\mathrm{R}$. Pal has shown in previous studies (Pal, 1996, 1998) where smaller droplets are shown to increase the viscosity. This might well be due to a modification of a yield stress which is not considered in these relative viscosity models. Therefore we would expect any variation of droplet sizes to be reflected in our value of the emulsion calibration parameter $r$.

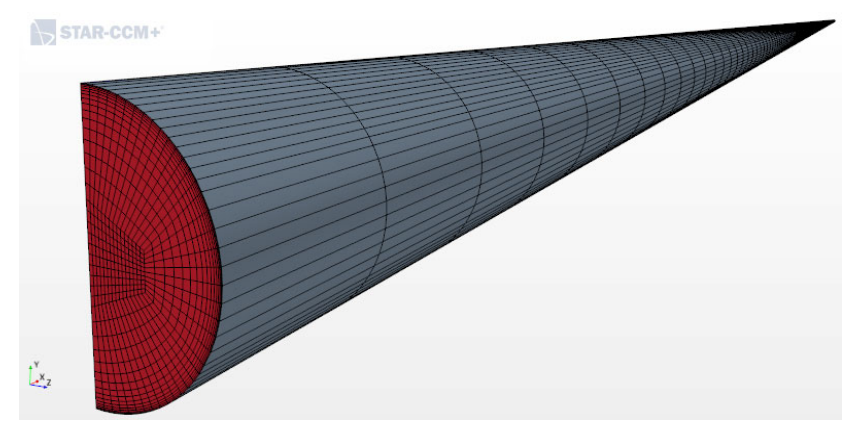

Fig. 18 3D half-pipe of 375,000 cells. 


\subsection{Simulation of the experiments}

Turbulence was modeled with the elliptic blending $k-\varepsilon$ model with the 2 nd order convection using the default parameters as outlined in Table 3 . We did not adjust any of these parameters in order to get a better match to data.

With such a wealth of pressure drop data given in Fig. 5 we needed to selectively choose which experiments to model due to limited computational resources and time. The set of data for the $32 \mathrm{~mm}$ ID pipe showed the largest influence of turbulence on the pressure drop Fig. 5(c) where a large peak in pressure drop is observed on the left hand side of the figure. The second peak is attributed to the emulsion rheology as the dispersion of particles come in contact and starts to interact with each other. The $32 \mathrm{~mm}$ ID pipe also has complete sets of data for all of the volume fractions and has the largest set of velocities studied.

In setting up the simulation we mirrored the dimensions of the experiments. The pipe was created with a mesh $15 \mathrm{~m}$ long and with surface (3D) or line probes (2D) at $12 \mathrm{~m}$ and $13.6 \mathrm{~m}$ from the inlet which were used to take the two values of pressure needed to calculate the pressure drop.

We simulated the $32 \mathrm{~mm}$ ID pipe in a $2 \mathrm{D}$ axisymmetric geometry at four velocities for this pipe, $U=0.5,1.8,2.0$ and $3.5 \mathrm{~m} \cdot \mathrm{s}^{-1}$. We simulated the lowest and highest velocities in

Table 3 Elliptic blending $k-\varepsilon$ parameters

\begin{tabular}{cc}
\hline Parameter & Value \\
\hline$C_{\mu}$ & 0.22 \\
$C_{1 e}$ & 1.44 \\
$C_{2 e}$ & 1.83 \\
$C_{3 e}$ & 1.0 \\
$C_{l}$ & 0.164 \\
$C_{\eta}$ & 75.0 \\
$C_{t}$ & 4.0 \\
$C_{T}$ & 1.0 \\
$C_{k}$ & 2.3 \\
$C_{1}$ & 1.7 \\
$C_{2}$ & 0.9 \\
$\sigma_{k}$ & 1.0 \\
$\sigma_{e}$ & 1.5 \\
$\sigma_{\phi}$ & 1.0 \\
$C_{M}$ (Sarkar) & 2.0 \\
\hline
\end{tabular}

Table 4 Parameters for emulsion rheology

\begin{tabular}{cc}
\hline$U\left(\mathrm{~m} \cdot \mathrm{s}^{-1}\right)$ & $r$ \\
\hline 0.5 & 1.7 \\
1.8 & 1.5 \\
2.0 & 1.7 \\
3.5 & 1.8 \\
\hline
\end{tabular}

3D $U=0.5$ and $3.5 \mathrm{~m} \cdot \mathrm{s}^{-1}$. All simulations used the inversion volume fraction of $\phi_{1}=0.84$ and inversion maximum packing of $\phi_{m i}=0.7, r_{i}=1.7$. The exact value of the inversion point could take larger values up to 0.9 as described in the experimental section, but we chose $\phi_{i}=0.84$ as it was guaranteed not to over-predict the pressure for the largest values of $\phi>0.9$ which are clearly inverted.

The only parameter which was varied to fit the pressure drop data was the emulsion calibration coefficient $r$, displayed in Table 4. If we needed to increase the mixture viscosity we decreased the value of $r$. For all of the simulations in this pipe we found $r=1.7$ captured the majority of the data. The lowest value used was 1.5 (for $U=1.8 \mathrm{~m} \cdot \mathrm{s}^{-1}$ ) and the highest was 1.8 (for $U=3.5 \mathrm{~m} \cdot \mathrm{s}^{-1}$ ). Decreasing $r$ makes the droplets more solid-like, recalling that a value of $r=1$ regains the original Krieger and Dougherty model for a suspension of hard solid spheres. The variation of this parameter helped to fit the highest data-point of the pressure drop corresponding to the laminar flows at a high volume fraction of water just before phase inversion around $\phi=0.83$. The reason for this variation could be due to variations of droplet size and distribution as discussed above.

Note that in our stress formulation using Eqs. (12) and (13) we did not include the particle pressure-like term which we used in the modeling of suspensions using the Morris and Boulay-type models (Morris and Boulay, 1999; Inkson et al., 2017). The presence of this term gives shear-induced particle migration for which we see no evidence in these experiments due to the homogeneous volume fraction observed. We found that this negative pressure term offset the value of the total pressure drop in the pipe. It is unclear if this particle pressure is needed in modeling these stabilized emulsions so we opted for the simpler model where the effect on the pressure is dominated by the shear viscosity alone. However we might want to include this effect in future work.

In the 3D pipe with low volume fraction of water cases, ( $\left.\phi=\alpha_{w}=0.09,0.19,0.29\right)$ we see some separation with the oil rising to the top of the pipe and forming a layer and the water sinking to the bottom, but by $\phi=\alpha_{w}=0.39$ and above we see a flat homogeneous profile (Fig. 19) and no separation of the phases is seen apart from at the cell next to the wall. In the bulk flow the large drag between the droplets and the high viscosity of the mixture tends to ensure that the phases do not separate. For the axisymmetric runs we have homogeneous volume fraction in all cases as gravity is neglected.

In order to determine if the cases were laminar or turbulent we evaluated $R e$ for the respective cases and identified which curve each data-point belonged to as described in Section 2.5.1. For the low velocity case we ran the three cases for $\phi<0.3$ as turbulent and the rest laminar. 
Figure 20(a) shows a comparison between the experimental data axisymmetric simulation and $3 \mathrm{D}$ simulation with gravity for the velocity $U=0.5 \mathrm{~m} \cdot \mathrm{s}^{-1}$. We see a very good agreement with the data, at this low velocity for all of the data points; however, we note that the $3 \mathrm{D}$ simulations

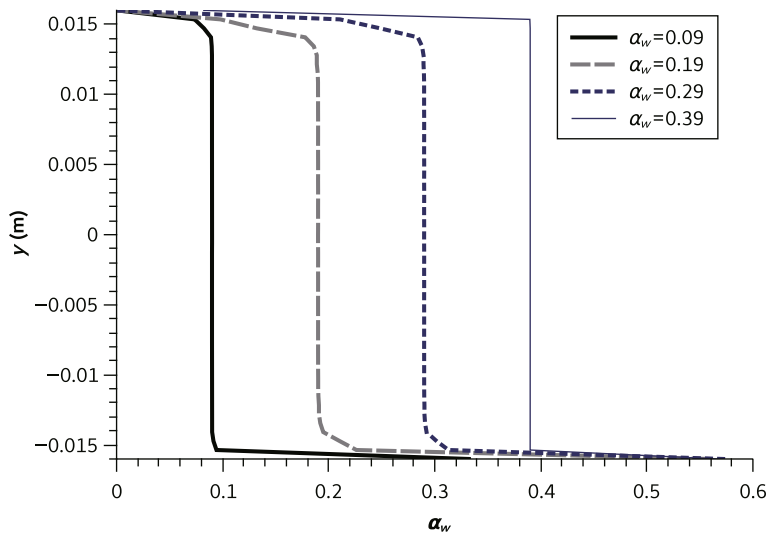

Fig. 19 Volume fraction of water profile down a vertical line at the outlet of the 3D $32 \mathrm{~mm}$ ID pipe with mixture velocity $0.5 \mathrm{~m} \cdot \mathrm{s}^{-1}$.

(a)

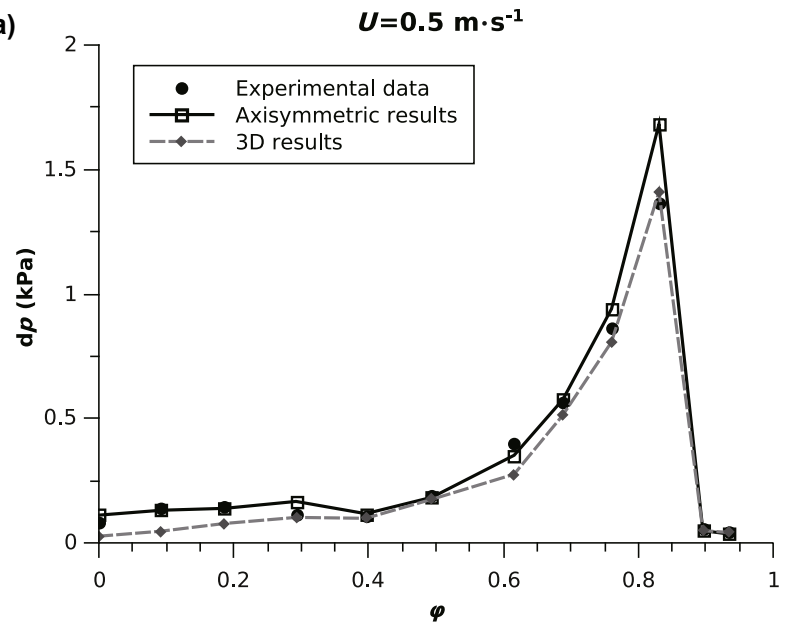

(b)

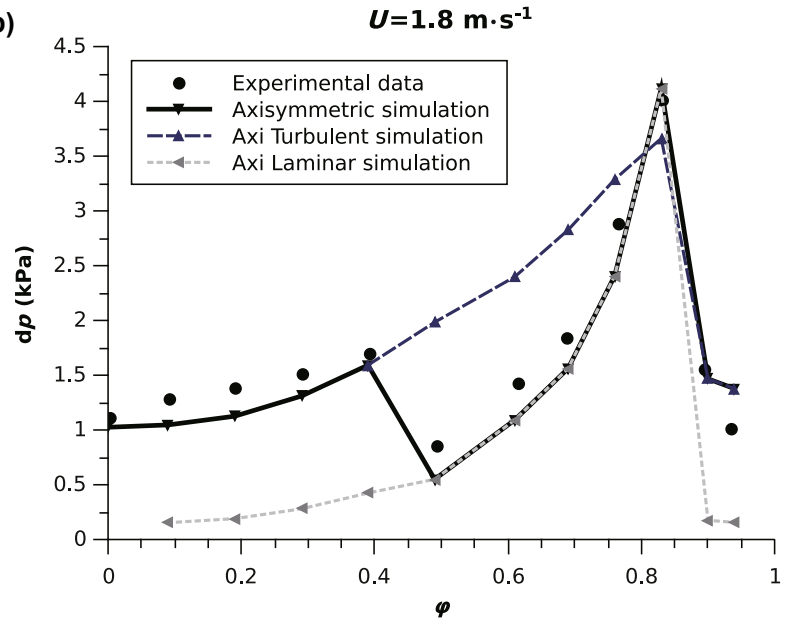

Fig. 20 Pressure drop as a function of water volume fraction for the $32 \mathrm{~mm}$ diameter pipe with mixture velocity (a) $0.5 \mathrm{~m} \cdot \mathrm{s}^{-1}$ and (b) $1.8 \mathrm{~m} \cdot \mathrm{s}^{-1}$. under-predict the data at the low volume fractions. This might be because of the presence of pure phases at the top (oil) and the bottom (water) of the pipe that exist in the 3D simulations. Figure 20(b) shows axisymmetric results for the velocity of $1.8 \mathrm{~m} \cdot \mathrm{s}^{-1}$ here the first five data-points display a large effect of turbulence, then the flow laminarizes at $\phi=0.49$, the rest of the results are laminar up to the peak where the flow then inverts and turbulent results are shown for the last two points $\phi=0.9,0.94$ although the last data point exists below the turbulent prediction which might reflect that the experiments showed signs of multiple emulsions (see Section 4.9). We ran additional simulations to cover the regions not seen in the experiments, by forcing the simulations to follow either the laminar or turbulent set-up for all of the water volume fractions. Through this additional data we clearly see that there exists two curves: a lower laminar curve entirely governed by emulsion rheology and a higher curve that is the combined result of emulsion rheology and turbulence eddy viscosity of the two phases, although we see curiously that the force turbulent peak at $\phi=0.83$ lies under the laminar result.

As the results are shown for a constant diameter pipe, the flow will always laminarize when the emulsion relative viscosity becomes high enough to lower $R e$ into the laminar regime.

At a velocity of $U=2.0 \mathrm{~m} \cdot \mathrm{s}^{-1}$ Fig. 21(a) shows similar results to $U=1.8 \mathrm{~m} \cdot \mathrm{s}^{-1}$ except that the sixth data point from the left corresponding to a volume fraction of dispersed water of $\phi=0.494$ is now a turbulent point which explains the noticeable gap between the curves in Fig. 5(c) that we were keen to replicate.

Results for the higher velocity of $U=3.5 \mathrm{~m} \cdot \mathrm{s}^{-1}$ are shown in Fig. 21(b). Here we again show the theoretical curves for pure laminar simulation and pure turbulent simulation. The data generally agrees with these curve where turbulence points are used for $\phi<0.68$ and $\phi>0.82$ and the rest lie on the laminar curve; however the point for $\phi=0.616$ is clearly in the laminar-turbulent transition regime. The high velocity resulted in a much larger discrepancy between axisymmetric and three-dimensional results, with the $3 \mathrm{D}$ pure turbulent results predicting a much larger pressure drop. At the highest peak point at $\phi=0.83$ we see that for the turbulent curve axisymmetric runs are unable to predict a higher value of pressure drop than the laminar result. So clearly the smaller, coarser mesh negatively affects the results for these fast, highly turbulent cases. However we are forcing a larger Reynolds number than the mesh allows and the simulations actually seem to laminarize themselves.

Figure 22 shows that the axisymmetric turbulent case of $U=3.5 \mathrm{~m} \cdot \mathrm{s}^{-1}, \alpha_{w}=0.49$ that the mixture eddy turbulent viscosity defined as $\mu_{m}^{t}=\alpha_{w} \mu_{w}^{t}+\alpha_{o} \mu_{o}^{t}$, is zero at the wall but grows in magnitude towards the center of the pipe. 
(a)

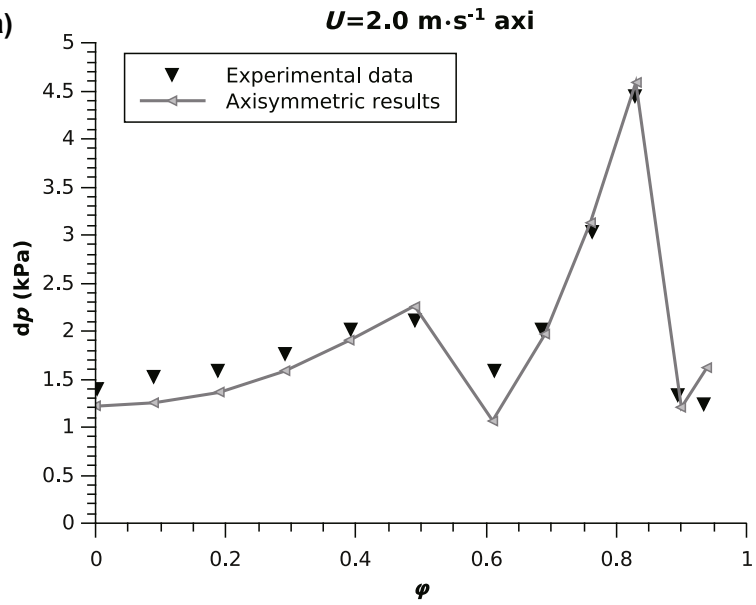

(b)

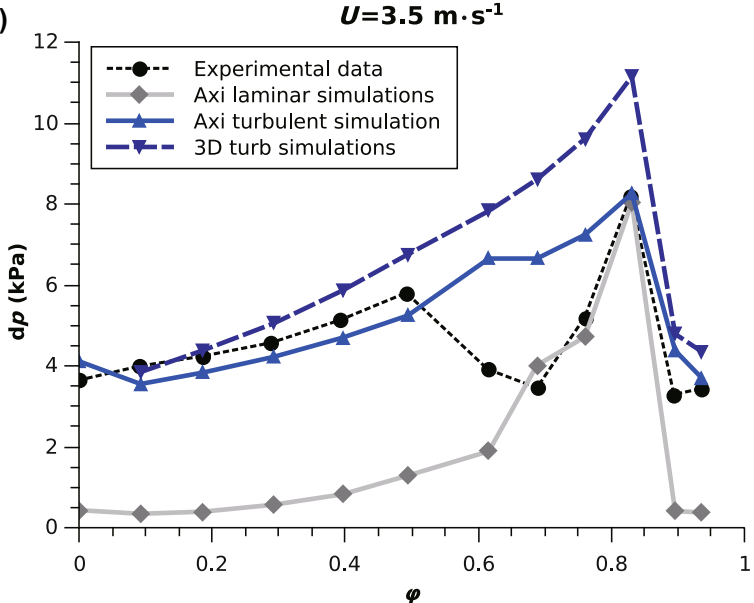

Fig. 21 Pressure drop as a function of water volume fraction for the $32 \mathrm{~mm}$ diameter pipe with mixture velocity (a) $2.0 \mathrm{~m} \cdot \mathrm{s}^{-1}$ and (b) $3.5 \mathrm{~m} \cdot \mathrm{s}^{-1}$.

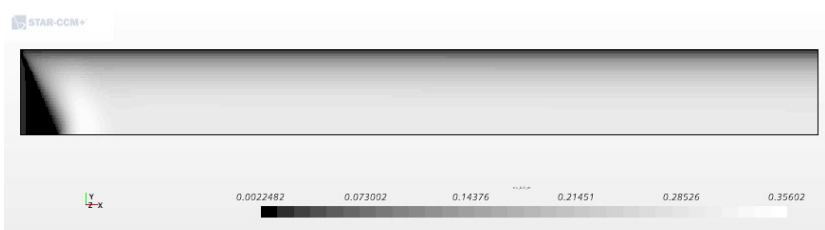

Fig. 22 Mixture turbulent viscosity in the axisymmetric $32 \mathrm{~mm}$ ID pipe with mixture velocity $3.5 \mathrm{~m} \cdot \mathrm{s}^{-1}, \alpha_{w}=0.49$. The figure is scaled 100 times in the $y$ direction with the inlet on the left.

By taking the average value of the turbulent viscosity over the area at the outlet we can see the relative contributions from the total, mixture, and turbulent viscosity in Fig. 23. Clearly the magnitude of the turbulent viscosity dominates over the mixture viscosity in turbulent cases however as seen in Fig. 22 the turbulent viscosity fades to zero at the wall where the laminar boundary layer exists, yet the mixture viscosity is uniform. As pressure drop is determined largely by the wall shear stress equation (1) then we can understand that the mixture viscosity becomes more relevant, especially when it exponentially increases towards the maximum packing asymptote of $r[\eta] \phi_{m}$ (see Section 2.1).

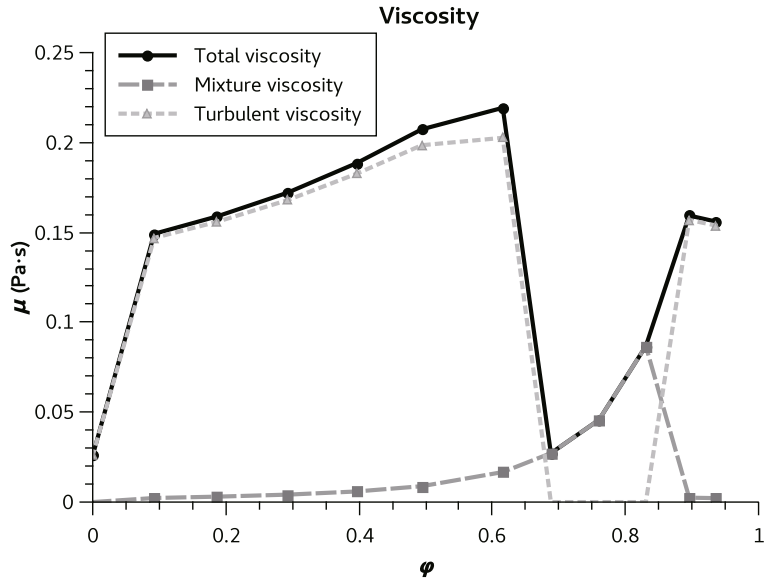

Fig. 23 Total, mixture, and turbulent viscosity average at the outlet of the axisymmetric $32 \mathrm{~mm}$ ID pipe with mixture velocity $3.5 \mathrm{~m} \cdot \mathrm{s}^{-1}, \alpha_{w}=0.49$.

The modeling did not require a shear-thinning effect which has also been observed in stabilized emulsions from the experimental data (Lee et al., 1997; Jansen et al., 2001). Although if shear-thinning was present we might expect that our $r$ parameter would actually have a lower value nearer to one, and that the thinning effect would reduce the relative viscosity in the steady flow state. Without further data from rheometry it was not possible for us to ascertain if shear-thinning was present in this set of data. However to model shear-thinning effects we would have to introduce a much more complex model with many more parameters that would have to be determined and fitted.

This approach is simple in that there is no effect of particle size on the results, which could be a factor when the volume fractions approach the jamming limit which has been experimentally shown by Pal $(1996,1998)$, which in turn could be due to the appearance of a yield stress as shown by Mason (1999). However the form of the Krieger and Dougherty relative viscosity model has a divergence to infinity at the maximum packing volume fraction which implies the presence of a yield stress which is beyond the scope of these simple viscosity models.

\section{Conclusions}

This study of surfactant stabilized has been performed for emulsions in four different pipe diameters. It was observed that even in the larger pipes as the water fraction was increased an initial turbulent flow condition sooner or later became laminar due to the higher effective viscosity of the emulsion. The transition from turbulent to laminar flow was observed in all pipe diameters (no turbulent flow at inversion was possible). A stable water-in-oil emulsion was established when increasing the water fraction starting from single phase oil up to the inversion point. No signs 
of multiple emulsions were seen before inversion. After inversion a multiple emulsion that consisted in droplets of water-in-oil emulsion flowing in the water continuous phase was observed which lead to reduced pressure drops. These multiple emulsions were unstable and quickly segregated under quiescent conditions. Water-in-oil emulsions were obtained up to $90 \%$ water cut. Increasing the water cut further resulted in a water continuous multiple emulsion. Interestingly phase inversion also happened at a constant water fraction. This inversion process was observed to occur at $90 \%$ water cut and it was driven by an increase in the mixture velocity (shear rate). The initial water-in-oil emulsion became a water-in-oil in water emulsion by increasing the shear rate. Furthermore, the inversion process resulted to be reversible when reducing the mixture velocity presenting some hysteresis. Different flow regimes could be identified during this reversal process: homogeneously dispersed water-in-oil droplets in water, water-in-oil droplets in water and water, water-in-oil emulsion bubbles in water, and a stratified or eventually annular flow of a water-in-oil emulsion surrounded by water. This annular like flow configuration was more frequent in the 32 and $16 \mathrm{~mm}$ pipes while the stratified like flow regime was dominant in the 60 and $90 \mathrm{~mm}$ pipes. The droplet sizes were observed to increase in size when increasing the water cut before the occurrence of inversion. The mixture velocity was also observed to have an impact on the droplet sizes. Higher mixture velocities resulted in smaller droplet sizes in all pipe diameters. Also, after inversion droplet sizes were smaller than the ones observed before inversion. This is attributed to the fact that the larger droplets coalesce to form the new continuous phase. However, chord length measurements should not be assumed as conclusive in multiple emulsions applications.

The modeling of the relative viscosity required a simple modification to the Krieger and Dougherty model in order to allow phase inversion to fit the entire set of data which we fitted with only two parameters. Namely, the inversion volume fraction $\phi_{i}$ and the calibration coefficient $r$, the pre-factor that shifts the maximum packing limit for the particles to reflect the less hard sphere like behaviour of emulsion droplets. It's remarkable that such a wide range of results can be described with no additional modeling for turbulence effects beyond the standard Reynolds Averaged Navier-Stokes (RANS) turbulence model.

The simulation has revealed the presence of two distinct curves for the laminar emulsions and turbulent emulsion pressure drops which serves to explain the sudden jump to a lower pressure drop curve in the transition from turbulent to laminar flow. The Reynolds number attributed to the constant pipe geometry changes according to the mixture viscosity which can act to laminarize even a very high speed flow when surfactant stabilized droplets are behaving similarly to solid particles resulting in an enhanced viscosity at high water volume fractions.

In future work we will investigate the effect of putting the extra mixture viscosity into the dynamic viscosity used within the turbulence models to see if this acts to relaminarize the flow automatically.

\section{Acknowledgements}

Jose Plasencia's work was funded by the Multiphase Flow Assurance Innovation Centre (FACE) - a research cooperation between Norwegian Universities and industry involving IFE, NTNU, and SINTEF. The centre was funded by The Research Council of Norway and industrial partners. The authors gratefully acknowledge Bjørnar Pettersen and Equinor for the FBRM probe. Nathanael Inkson would like to thank Dr. Mohit Tandon for building the emulsion model upon his multiphase code.

\section{Funding note}

Open access funding provided by NTNU Norwegian University of Science and Technology.

\section{Declaration of competing interest}

The authors have no competing interests to declare that are relevant to the content of this article.

\section{References}

Angeli, P., Hewitt, G. F. 1999. Pressure gradient in horizontal liquidliquid flows. Int J Multiphase Flow, 24: 1183-1203.

Arirachakaran, S., Oglesby, K. D., Malinowsky, M. S., Shoham, O., Brill, J. P. 1989. An analysis of oil/water flow phenomena in horizontal pipes. In: Proceedings of the SPE Production Operations Symposium, Paper No. SPE-18836-MS.

Bannwart, A. C., Rodriguez, O. M. H., de Carvalho, C. H. M., Wang, I. S., Vara, R. M. O. 2004. Flow patterns in heavy crude oil-water flow. J Energy Resour Technol, 126: 184-189.

Billard, F., Laurence, D. 2012. A robust $k-\varepsilon-\mathrm{v} 2^{-} / \mathrm{k}$ elliptic blending turbulence model applied to near-wall, separated and buoyant flows. Int J Heat Fluid Fl, 33: 45-58.

Boxall, J. A., Koh, C. A., Sloan, E. D., Sum, A. K., Wu, D. T. 2010. Measurement and calibration of droplet size distributions in water-in-oil emulsions by particle video microscope and a focused beam reflectance method. Ind Eng Chem Res, 49: 1412-1418.

Cengel, J. A., Faruqui, A. A., Finnigan, J. W., Wright, C. H., Kundsen, J. G. 1962. Laminar and turbulent flow of unstable liquid-liquid emulsions. AIChE J, 8: 335-339.

Durbin, P. A. 1993. A Reynolds stress model for near-wall turbulence. J Fluid Mech, 249: 465-498. 
Galindo-Alvarez, J., Sadtler, V., Choplin, L., Salager, J. L. 2011. Viscous oil emulsification by catastrophic phase inversion: Influence of oil viscosity and process conditions. Ind Eng Chem Res, 50: 5575-5583.

Groeneweg, F., Agterof, W. G. M., Jaeger, P., Janssen, J. J. M., Wieringa, J. A., Klahn, J. K. 1998. On the mechanism of the inversion of emulsions. Chem Eng Res Des, 76: 55-63.

Inkson, N. J., Papoulias, D., Tandon, M., Reddy, V., Lo, S. 2017. An Eulerian-Eulerian formulation of suspension rheology using the finite volume method. J Non-Newton Fluid Mech, 245: 38-48.

Inkson, N. J., Plasencia, J., Lo, S. 2014. Predicting emulsion pressure drop in pipes through CFD multiphase rheology models. In: Proceedings of the 10th International Conference on CFD in Oil \& Gas, Metallurgical and Process Industries: 461-466.

Ioannou, K., Nydal, O. J., Angeli, P. 2005. Phase inversion in dispersed liquid-liquid flows. Exp Therm Fluid Sci, 29: 331-339.

Ishii, M., Hibiki, T. 2006. Thermo-Fluid Dynamics of Two-Phase Flow. Boston: Springer US.

Jansen, K. M. B., Agterof, W. G. M., Mellema, J. 2001. Viscosity of surfactant stabilized emulsions. J Rheol, 45: 1359-1371.

Jones, W. P., Launder, B. E. 1972. The prediction of laminarization with a two-equation model of turbulence. Int J Heat Mass Tran, 15: 301-314

Krieger, I. M., Dougherty, T. J. 1959. A mechanism for non-Newtonian flow in suspensions of rigid spheres. T Soc Rheol, 3: 137-152.

Lee, H. M., Lee, J. W., Park, O. O. 1997. Rheology and dynamics of water-in-oil emulsions under steady and dynamic shear flow. J Colloid Interf Sci, 185: 297-305.

Lee, J. D., So, J. H., Yang, S. M. 1999. Rheological behavior and stability of concentrated silica suspensions. J Rheol, 43: 1117-1140.

Lo, S. 2015. In: Industrial Computational Fluid Dynamics. Von Karman Institute for Fluid Dynamics.

Lovick, J., Angeli, P. 2004. Experimental studies on the dual continuous flow pattern in oil-water flows. Int J Multiphase Flow, 30: 139-157.

Maaß, S., Wollny, S., Voigt, A., Kraume, M. 2011. Experimental comparison of measurement techniques for drop size distributions in liquid/liquid dispersions. Exp Fluids, 50: 259-269.

Manceau, R., Hanjalić, K. 2002. Elliptic blending model: a new near-wall Reynolds-stress turbulence closure. Phys Fluids, 14: 744-754.

Mason, T. G. 1999. New fundamental concepts in emulsion rheology. Curr Opin Colloid In, 4: 231-238.

Morris, J. F., Boulay, F. 1999. Curvilinear flows of noncolloidal suspensions: The role of normal stresses. J Rheol, 43: 1213-1237.

Nädler, M., Mewes, D. 1997. Flow induced emulsification in the flow of two immiscible liquids in horizontal pipes. Int J Multiphase Flow, 23: 55-68.

Pal, R. 1993. Pipeline flow of unstable and surfactant-stabilized emulsions. AIChE J, 39: 1754-1764.

Pal, R. 1996. Effect of droplet size on the rheology of emulsions. AIChE $J, 42: 3181-3190$

Pal, R. 1998. Effects of droplet size and droplet size distribution on the rheology of oil- in-water emulsions. In: Proceedings of the 7th UNITAR International Conference for Heavy Crude and Tar Sands.

Pal, R. 2007. Mechanism of turbulent drag reduction in emulsions and bubbly suspensions. Ind Eng Chem Res, 46: 618-622.
Patankar, S. V. 1990. Numerical Heat Transfer and Fluid Flow. Taylor \& Francis.

Patankar, S. V., Spalding, D. B. 1972. A calculation procedure for heat, mass and momentum transfer in three-dimensional parabolic flows. Int J Heat Mass Tran, 15: 1787-1806.

Piela, K., Delfos, R., Ooms, G., Westerweel, J., Oliemans, R. V. A., Mudde, R. F. 2006. Experimental investigation of phase inversion in an oil-water flow through a horizontal pipe loop. Int J Multiphase Flow, 32: 1087-1099.

Plasencia, J., Nydal, O. J. 2010. Influence of the pipe diameter in dispersed oil-water flows. In: Proceedings of the 7th International Conference on Multiphase Flow.

Plasencia, J., Pettersen, B., Nydal, O. J. 2013. Pipe flow of water-incrude oil emulsions: Effective viscosity, inversion point and droplet size distribution. J Petrol Sci Eng, 101: 35-43.

Richardson, J. F., Zaki, W. N. 1997. Sedimentation and fluidisation: Part I. Chem Eng Res Des, 75: S82-S100.

Rusche, H., Issa, R. I. 2000. The effect of voidage on the drag force on particles, droplets and bubbles in dispersed two-phase flow. In: Proceedings of the Japanese European Two-Phase Flow Meeting.

Salager, J. L., Márquez, L., Peña, A. A., Rondón, M., Silva, F., Tyrode, E. 2000. Current phenomenological know-how and modeling of emulsion inversion. Ind Eng Chem Res, 39: 2665-2676.

Sarkar, S., Balakrishnan, L. 1990. Application of a Reynolds stress turbulence model to the compressible shear layer. In: Proceedings of the 21st Fluid Dynamics, Plasma Dynamics and Lasers Conference.

Schiller, L., Naumann, Z. 1935. A drag coefficient correlation. VDI Zeitung, 77: 318-320.

Siemens Digital Industries Software (SDIS). 2020. Simcenter3D STAR-CCM+ documentation. Available at https://www.plm.automation.siemens.com/global/en/products/ simcenter/STAR-CCM.html.

Tyrode, E., Mira, I., Zambrano, N., Márquez, L., Rondón-Gonzalez, M., Salager, J. L. 2003. Emulsion catastrophic inversion from abnormal to normal morphology. 3. conditions for triggering the dynamic inversion and application to industrial processes. Ind Eng Chem Res, 42: 4311-4318.

Vasquez, S. A., Ivanov, V. A. 2000. A phase coupled method for solving multiphase problems on unstructured meshes. In: Proceedings of the ASME FEDSM: 734-748.

Open Access This article is licensed under a Creative Commons Attribution 4.0 International License, which permits use, sharing, adaptation, distribution and reproduction in any medium or format, as long as you give appropriate credit to the original author(s) and the source, provide a link to the Creative Commons licence, and indicate if changes were made.

The images or other third party material in this article are included in the article's Creative Commons licence, unless indicated otherwise in a credit line to the material. If material is not included in the article's Creative Commons licence and your intended use is not permitted by statutory regulation or exceeds the permitted use, you will need to obtain permission directly from the copyright holder.

To view a copy of this licence, visit http://creativecommons.org/licenses/by/4.0/. 